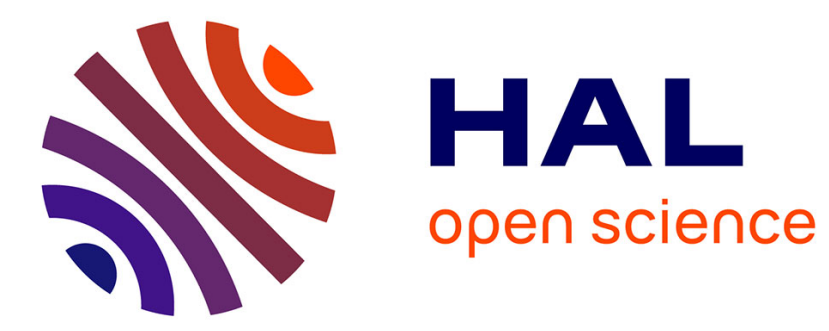

\title{
The reverse mathematics of the Thin set and the Erdos-Moser theorems
}

Lu Liu, Ludovic Patey

\section{To cite this version:}

Lu Liu, Ludovic Patey. The reverse mathematics of the Thin set and the Erdos-Moser theorems. The Journal of Symbolic Logic, 2022, 87 (1), pp.313-346. 10.1017/jsl.2021.98 . hal-03362591

\section{HAL Id: hal-03362591 \\ https://hal.science/hal-03362591}

Submitted on 1 Oct 2021

HAL is a multi-disciplinary open access archive for the deposit and dissemination of scientific research documents, whether they are published or not. The documents may come from teaching and research institutions in France or abroad, or from public or private research centers.
L'archive ouverte pluridisciplinaire HAL, est destinée au dépôt et à la diffusion de documents scientifiques de niveau recherche, publiés ou non, émanant des établissements d'enseignement et de recherche français ou étrangers, des laboratoires publics ou privés. 


\title{
THE REVERSE MATHEMATICS OF THE THIN SET AND THE ERDÖS-MOSER THEOREMS
}

\author{
LU LIU AND LUDOVIC PATEY
}

\begin{abstract}
The thin set theorem for $n$-tuples and $k$ colors $\left(\mathrm{TS}_{k}^{n}\right)$ states that every $k$-coloring of $[\mathbb{N}]^{n}$ admits an infinite set of integers $H$ such that $[H]^{n}$ avoids at least one color. In this paper, we study the combinatorial weakness of the thin set theorem in reverse mathematics by proving neither $\mathrm{TS}_{k}^{n}$, nor the free set theorem $\left(\mathrm{FS}^{n}\right)$ imply the Erdős-Moser theorem (EM) whenever $k$ is sufficiently large (answering a question of Patey and giving a partial result towards a question of Cholak Giusto, Hirst and Jockusch). Given a problem P, a computable instance of $\mathrm{P}$ is universal iff its solution computes a solution of any other computable P-instance. It has been established that most of Ramseytype problems do not have a universal instance, but the case of Erdös-Moser theorem remained open so far. We prove that Erdős-Moser theorem does not admit a universal instance (answering a question of Patey).
\end{abstract}

\section{INTRODUCTION}

In this paper, we study the computability-theoretic strength of Ramsey's theorem when we weaken the notion of homogeneous set by allowing more colors. In particular, we show that this variation yields a strong weakening of the statement which is impacted in reverse mathematics.

Reverse mathematics is a foundational program which seeks to determine the optimal axioms to prove "ordinary" theorems 4]. It uses the framework of subsystems of second-order arithmetic, with a base theory called $\mathrm{RCA}_{0}$, informally capturing "computable mathematics". When the first-order part consists of the standard integers, the models of RCA 0 are fully specified by their second-order parts, which are precisely the Turing ideals. A Turing ideal $\mathcal{I}$ is a collection of sets which is closed downward under the Turing reduction $(\forall X \in \mathcal{I})\left(\forall Y \leq_{T} X\right) Y \in \mathcal{I}$ and closed under the effective join $(\forall X, Y \in \mathcal{I}) X \oplus Y \in \mathcal{I}$. We shall only consider such models, called $\omega$-models.

The early study of reverse mathematics has seen the emergence of 4 subsystems of second-order arithmetic linearly ordered by provability relation, such that most of the ordinary theorems are either provable in $R C A_{0}$, or equivalent in $R C A_{0}$ to one of them. These subsystems, together with $\mathrm{RCA}_{0}$, form the "Big Five" 11]. Among them, let us mention ACA, standing for arithmetical comprehension axiom, and WKL, standing for weak König's lemma, which states that every infinite binary tree has an infinite path. See Simpson [20] for a good introduction to reverse mathematics. Among the theorems studied in reverse mathematics, Ramsey's theorem plays an important role, since Ramsey's theorem for pairs is historically the first example of statement which does not satisfy this empirical observation.

Definition 1.1 (Ramsey's theorem). A subset $H$ of $\omega$ is homogeneous for a coloring $f:[\omega]^{n} \rightarrow k$ (or $f$-homogeneous) if each $n$-tuple over $H$ is given the same 
color by $f$. $\mathrm{RT}_{k}^{n}$ is the statement "Every coloring $f:[\omega]^{n} \rightarrow k$ has an infinite $f$-homogeneous set".

$\mathrm{RT}_{k}^{1}$ is nothing but the infinite pigeonhole principle which is provable in $\mathrm{RCA}_{0}$. Jockusch [6] (see Simpson [20]) proved that $\mathrm{RT}_{k}^{n}$ is equivalent to ACA over $\mathrm{RCA}_{0}$ whenever $n \geq 3$, and that WKL does not imply $\mathrm{RT}_{2}^{2}$ over $\mathrm{RCA}_{0}$. The computabilitytheoretic strength of Ramsey's theorem for pairs was unknown for a long time, until Seetapun [19] proved that $\mathrm{RT}_{2}^{2}$ does not imply ACA over $\mathrm{RCA}_{0}$, and that the first author 9] proved that $\mathrm{RT}_{2}^{2}$ does not imply WKL over $\mathrm{RCA}_{0}$, thereby showing that $\mathrm{RT}_{2}^{2}$ is not even linearly ordered with the Big Five.

This analysis of Ramsey's theorem naturally started new research axis, among which the search for weakenings of Ramsey's theorem for arbitrary $n$-tuples which would not imply ACA. Ramsey's theorem can be seen as a problem, whose instances are $k$-colorings of $[\omega]^{n}$, and whose solutions are infinite homogeneous sets. This problem has two explicit parameters, namely, the size $n$ of the $n$-tuples, and the number $k$ of colors of the coloring. There is one implicit parameter which is the number of colors $\ell$ allowed in the solution. In the case of a homogeneous set, $\ell=1$. In this paper, we give a partial answer to the following question.

How does the number of colors allowed in a solution impact the computability-theoretic strength of Ramsey's theorem?

We are in particular interested in the case where $\ell=k-1$. This yields the notion of thin set.

Definition 1.2 (Thin set theorem). Given a coloring $f:[\omega]^{n} \rightarrow k$ (resp. $f:[\omega]^{n} \rightarrow$ $\omega$ ), a set $H$ is thin for $f$ (or $f$-thin) if $\left|f\left([H]^{n}\right)\right| \leq k-1$ (resp. $f\left([H]^{n}\right) \neq \omega$ ). For every $n \geq 1$ and $k \geq 2, \operatorname{TS}_{k}^{n}$ is the statement "Every coloring $f:[\omega]^{n} \rightarrow k$ has an infinite $f$-thin set" and $\mathrm{TS}_{\omega}^{n}$ is the statement "Every coloring $f:[\omega]^{n} \rightarrow \omega$ has an infinite $f$-thin set".

In particular, $\mathrm{TS}_{2}^{n}$ is Ramsey's theorem for $n$-tuples and 2 colors. The thin set theorem was introduced in reverse mathematics by Friedman [3] and studied by Cholak, Giusto, Hirst and Jockusch 2]. Wang 21] proved the surprising result that for every $n \geq 1$ and every sufficiently large $k$ (with an explicit upper bound on $k), \mathrm{TS}_{k}^{n}$ does not imply ACA, thereby showing that allowing more colors in the solutions yields a strict weakening of Ramsey's theorem, which is already reflected in reverse mathematics. The second author refined Wang's analysis by proving that $\mathrm{TS}_{k}^{n}$ implies neither WKL 13, nor $\mathrm{TS}_{\ell}^{m}$ for every $n$ and sufficiently large $k$ 18. In particular, the statement $(\forall n)(\exists k) \mathrm{TS}_{k}^{n}$ does not imply $\mathrm{RT}_{2}^{2}$ over $\mathrm{RCA}_{0}$. In this paper, we answer the following sub-question.

What consequences of Ramsey's theorem for pairs are already consequences of the thin set theorem in reverse mathematics?

The thin set theorem for pairs with $\omega$ colors $\left(\mathrm{TS}_{\omega}^{2}\right)$ seems combinatorially very weak, however, it has a diagonalization power similar to Ramsey's theorem for pairs. For example, there is a computable instance of $\mathrm{TS}_{\omega}^{2}$ with no $\Sigma_{2}^{0}$ solution [2], and given any low 2 set $X$, there is a computable instance of $\mathrm{TS}_{\omega}^{2}$ with no $X$-computable solution [14]. One can strengthen the thin set theorem by asking, given a coloring $f:[\omega]^{n} \rightarrow \omega$, for an infinite set $H$ such that $H \backslash\{x\}$ is $f$-thin for every $x \in H$. This yields the notion of free set. 
Definition 1.3 (Free set theorem). Given a coloring $f:[\omega]^{n} \rightarrow \omega$, a set $H$ is free for $f$ (or $f$-free) if for every $\sigma \in[H]^{n}, f(\sigma) \in H \rightarrow f(\sigma) \in \sigma$. For every $n \geq 1$, FS $^{n}$ is the statement "Every coloring $f:[\omega]^{n} \rightarrow \omega$ has an infinite $f$-free set".

The free set theorem is usually studied together with the thin set theorem, as both statements are combinatorially very close. The standard proof of $\mathrm{FS}^{n}$ involves the statement $(\exists k) \mathrm{TS}_{k}^{n}$ in a way that propagates most of the computabilitytheoretic properties of the thin set theorem to the free set theorem. This is why any known proof that $(\exists k) \mathrm{TS}_{k}^{n}$ does not imply another statement $\mathrm{P}$ over $\mathrm{RCA}_{0}$ empirically yields a proof that $\mathrm{FS}^{n}$ does not imply P [2, 21, 18, 13. This will again be the case in our paper.

1.1. Main results. Ramsey's theorem for pairs admits various decompositions, that is, is equivalent to the conjunction of strictly weaker statements. Among them, the decomposition into the Erdös-Moser theorem and the Ascending Descending sequence principle is particularly interesting for various technical reasons.

Definition 1.4 (Erdős-Moser theorem). A tournament $T$ is an irreflexive binary relation such that for all $x, y \in \omega$ with $x \neq y$, exactly one of $T(x, y)$ or $T(y, x)$ holds. A set $H$ is $T$-transitive if the relation $T$ over $H$ is transitive in the usual sense. EM is the statement "Every infinite tournament $T$ has an infinite transitive subtournament."

Definition 1.5 (Ascending descending sequence). Given a linear order $\left(L,<_{L}\right)$, an ascending (descending) sequence is a set $S$ such that for every $x<_{\mathbb{N}} y \in S$, $x<_{L} y\left(x>_{L} y\right)$. ADS is the statement "Every infinite linear order admits an infinite ascending or descending sequence".

Bovykin and Weiermann [1] proved Ramsey's theorem for pairs as follows: Given a coloring $f:[\mathbb{N}]^{2} \rightarrow 2$, we can see $f$ as a tournament $T$ such that whenever $x<_{\mathbb{N}} y$, $T(x, y)$ holds if and only if $f(x, y)=1$. Any $T$-transitive set $H$ can be seen as a linear order $(H, \prec)$ such that for every $x<_{\mathbb{N}} y: x \prec y$ if and only if $f(x, y)=1$. Any infinite ascending or descending sequence is $f$-homogeneous. It is therefore natural to study the ascending descending sequence principle together with the Erdős-Moser theorem. Lerman, Solomon and Towsner [8] proved that EM does not imply ADS over RCA 0 , while Hirschfeldt and Shore [5] proved that ADS does not imply EM. The second author asked [18] whether any of $\mathrm{FS}^{2}, \mathrm{TS}_{\omega}^{2}$, or $\mathrm{TS}_{3}^{2}$ implies EM over RCA $\mathrm{R}_{0}$. We answer this question negatively.

Theorem 1.6. Over $\mathrm{RCA}_{0}$, $\mathrm{WKL}+\mathrm{COH}+\mathrm{TS}_{4}^{2}+(\forall n)(\exists k) \mathrm{TS}_{k}^{n}+(\forall n) \mathrm{FS}^{n}$ implies none of SEM, STS $S_{3}^{2}$ and SADS.

In Theorem 1.6, $\mathrm{STS}_{k}^{2}$, SEM and SADS denote the restriction of $\mathrm{TS}_{k}^{2}$, EM and ADS to stable colorings, respectively. A coloring $f:[\omega]^{2} \rightarrow k$ is stable if for every $x \in \omega, \lim _{s} f(x, s)$ exists. In the case of SADS, this yields the statement "Every linear order of type $\omega+\omega^{*}$ has an infinite ascending or descending sequence." $\mathrm{COH}$ is the statement "For every sequence of sets $\vec{R}=\left(R_{0}, R_{1}, \ldots\right)$, there is an infinite set $C$ such that $C \subseteq^{*} R_{i}$ or $C \subseteq \subseteq^{*} \bar{R}_{i}$ for every $i \in \omega$." Such set $C$ is called $\vec{R}$-cohesive

The separation result, Theorem 1.6. shows that although the thin set theorem shares many lower bounds with Ramsey's theorem, allowing more colors in the solutions yields a statement with strictly weaker computability-theoretic properties. 
Cholak, Giusto, Hirst and Jockusch 2 Question 7.4 asked whether FS ${ }^{2}+$ CAC implies $\mathrm{RT}_{2}^{2}$. Bovykin and Weiermann [1] proved that $\mathrm{RT}_{2}^{2}$ is equivalent to $\mathrm{EM}+$ ADS. It's well known CAC implies ADS. Thus Theorem 1.6 (FS ${ }^{2}$ does not imply EM) is a partial result towards a negative answer to Cholak, Giusto, Hirst and Jockusch's question.

For a problem $\mathrm{P}$, a computable $\mathrm{P}$-instance $I^{*}$ is universal iff for every computable P-instance $I$, every solution of $I^{*}$ computes a solution of $I$. The most well known example of universal instance is for WKL, there is a computable tree $T^{*} \subseteq 2^{<\omega}$ so that every infinite path through $T^{*}$ is of PA degree. Thus every infinite path through $T^{*}$ computes an infinite path of a given computable infinite tree $T$. Patey [14] systematically studied which Ramsey type problem admits universal instance. For many Ramsey type problems, this is now clear that they don't admit universal instance. As many cases in logic, usually, if a problem admits universal instance, it is relatively easy to construct one (coding is usually not hard when exist). For several problems, the question remains. The second author asked in [15] that whether EM admits a universal instance. We answer this question negatively.

Theorem 1.7. EM does not have universal instance.

The first author asked a similar question with respect to arbitrary instance of $\mathrm{RT}_{2}^{1}$. Clearly, an instance is universal means it encode information of any other computable instance. For a problem $\mathrm{P}$, we consider the mass problem generated by instances of P. For P-instances $I, \hat{I}$ (not necessarily computable), we say $I$ encode $\hat{I}$ iff every solution of $I$ computes a solution of $\hat{I}$. i.e., the set of solutions of $\hat{I}$ is Muchnick reducible to that of $I$. Liu [10] asked whether there is a $\mathrm{RT}_{2}^{1}$ instance $X$ that is maximal (in the lattice of encoding relation) in the sense that for every $\mathrm{RT}_{2}^{1}$ instance $Y$, if $Y$ encodes $X$, then $X$ encodes $Y$.

1.2. Organization. The paper is divided into two main sections, corresponding to the proofs of Theorem 1.6 and Theorem 1.7, respectively. In section 2, we introduce a framework of preservation of 2-hyperimmunity, and develop its basic properties in subsection 2.1. We then prove in subsection 2.3 that $\mathrm{TS}_{4}^{2}$ preserves 2-hyperimmunity, then generalize the proof to $(\forall n)(\exists k) \mathrm{TS}_{k}^{n}$ in subsection 2.5. We prove that $\mathrm{FS}^{2}$ preserves 2-hyperimmunity in subsection 2.6, and again generalize it to $(\forall n) \mathrm{FS}^{n}$ in subsection 2.7. In section 3, we prove Theorem 1.7 .

1.3. Notation. Given two sets $A$ and $B$, we write $A<B$ iff $x<y$ for all $x \in$ $A, y \in B(A<\emptyset$ and $\emptyset<B$ both hold); we write $A>y$ iff $x>y$ for all $x \in A$. We use $|A|$ to denote the cardinal of the set $A$. Denote by $[A]^{n}$ the collection of $n$-element subsets of $A ;[A]^{<\omega}$ the collection of finite subsets of $A$. Usually, we use $F, E, D$ and sometimes $\sigma, \tau$ to denote finite set of integers; $X, Y, Z$ to denote infinite set of integers; $A, B, H, G$ to denote set of integers.

A Mathias condition is a pair $(F, X)$ where $F$ is a finite set, $X$ is an infinite set. A condition $(E, Y)$ extends $(F, X)$ (written $(E, Y) \leq(F, X))$ if $E \supseteq F, E \backslash F>$ $F, E \backslash F \subseteq X, Y \subseteq X$. Note that we do not require $F<X$ for a condition $(F, X)$, but continuity is ensured by the extension relation. A set $G$ satisfies a Mathias condition $(F, X)$ if $F \subseteq G, G \backslash F \subseteq X, G \backslash F>F$. A Mathias condition $c$ is seen as the collection $\{G: G$ satisfies $(F, X)\}$; note that $c \leq d$ iff the collection of $c$ is a sub collection of $d$. We adopt the convention that if $\Phi^{F}(n) \downarrow$ and $G>F$, then $\Phi^{F \cup G}(n) \downarrow=\Phi^{F}(n)$. 
THE REVERSE MATHEMATICS OF THE THIN SET AND THE ERDŐS-MOSER THEOREMS5

\section{FREE AND THIN SETS WHICH ARE NOT TRANSITIVE}

This section is devoted to the proof of Theorem 1.6 that we recall now.

Theorem 1.6. Over $\mathrm{RCA}_{0}, \mathrm{WKL}+\mathrm{COH}+\mathrm{TS}_{4}^{2}+(\forall n)(\exists k) \mathrm{TS}_{k}^{n}+(\forall n) \mathrm{FS}^{n}$ implies none of SEM, STS 2 and SADS.

For this, we are going to prove that WKL, $\mathrm{COH}, \mathrm{TS}_{4}^{2}$ and $\mathrm{FS}^{n}$ preserve a computability-theoretic notion that we call 2-hyperimmunity, while none of SEM, $\mathrm{STS}_{3}^{2}$ and SADS does.

\section{Definition 2.1.}

(1) A bifamily is a collection $\mathcal{H}$ of ordered pairs of finite sets which is closed downward under the product subset relation, that is, such that if $(C, D) \in$ $\mathcal{H}$ and $E \subseteq C$ and $F \subseteq D$, then $(E, F) \in \mathcal{H}$.

(2) A biarray is a collection of finite sets $(\vec{E}, \vec{F})=\left\langle E_{n}, F_{n, m}: n, m \in \omega\right\rangle$ such that $E_{n}>n$ and $F_{n, m}>m$ for every $n, m \in \omega$. A biarray $(\vec{E}, \vec{F})$ meets a bifamily $\mathcal{H}$ if there is some $n, m \in \omega$ such that $\left(E_{n}, F_{n, m}\right) \in \mathcal{H}$.

(3) A bifamily $\mathcal{H}$ is $C$-2-hyperimmune if every $C$-computable biarray meets $\mathcal{H}$.

We shall relate the notion of 2-hyperimmunity and various notions of immunity in section 2.1. For notation convenience, in this section we regard each Turing machine $\Phi$ as computing a biarray. We will therefore assume that whenever $\Phi(n ; 1)$ converges, then it will output (the canonical index of) a finite set $E_{n}>n$ and whenever $\Phi(n, m ; 2)$ converges, then it will output a finite set $F_{n, m}>m$.

Definition 2.2. Fix a problem $P$.

(1) $\mathrm{P}$ preserves 2-hyperimmunity if for every bifamily $\mathcal{H}$ that is $C$-2-hyperimmune, every $C$-computable P-instance admit a solution $G$ such that $\mathcal{H}$ is $C \oplus G$ 2-hyperimmune.

(2) $\mathrm{P}$ strongly preserves 2-hyperimmunity if for every bifamily $\mathcal{H}$ that is $C$-2hyperimmune, every P-instance admit a solution $G$ such that $\mathcal{H}$ is $C \oplus G$ 2-hyperimmune.

The following lemma is a particular case of Lemma 3.4.2 in [17]. We reprove it for the sake of containment.

Lemma 2.3. If some problems $\mathrm{P}_{1}, \mathrm{P}_{2}, \ldots$ preserve 2-hyperimmunity while another problem $\mathrm{Q}$ does not, then the conjunction $\bigwedge_{i} \mathrm{P}_{i}$ does not imply $\mathrm{Q}$ over $\mathrm{RCA}_{0}$.

Proof. Since $\mathrm{Q}$ does not preserve 2-hyperimmunity, there is some set $C$, a bifamily $\mathcal{H}$ that is $C$-2-hyperimmune, and a $\mathrm{Q}$-instance $B$ such that for every solution $G$, $\mathcal{H}$ is not $C \oplus G$-2-hyperimmune. Since each $\mathrm{P}_{i}$ preserve 2-hyperimmunity, we can define an infinite sequence of sets $C=Z_{0} \leq_{T} Z_{1} \leq_{T} \ldots$ such that

(i) $\mathcal{H}$ is $Z_{n}$-2-hyperimmune for every $n$

(ii) For every $i, n \in \omega$, every $Z_{n}$-computable $\mathrm{P}_{i}$-instance has a $Z_{m}$-computable solution for some $m$

Consider the $\omega$-structure $\mathcal{M}=\left\{X:(\exists n) X \leq_{T} Z_{n}\right\}$. By construction, $B \in \mathcal{M}$, and by (i), $\mathcal{H}$ is $C \oplus G$-2-hyperimmune for every $G \in \mathcal{M}$. It follows that the Q-instance $B \in \mathcal{M}$ has no solution in $\mathcal{M}$, so $\mathcal{M} \not \models \mathrm{Q}$. By (ii), $\mathcal{M} \models \mathrm{P}_{i}$ for every $i \in \omega$. This completes the proof. 


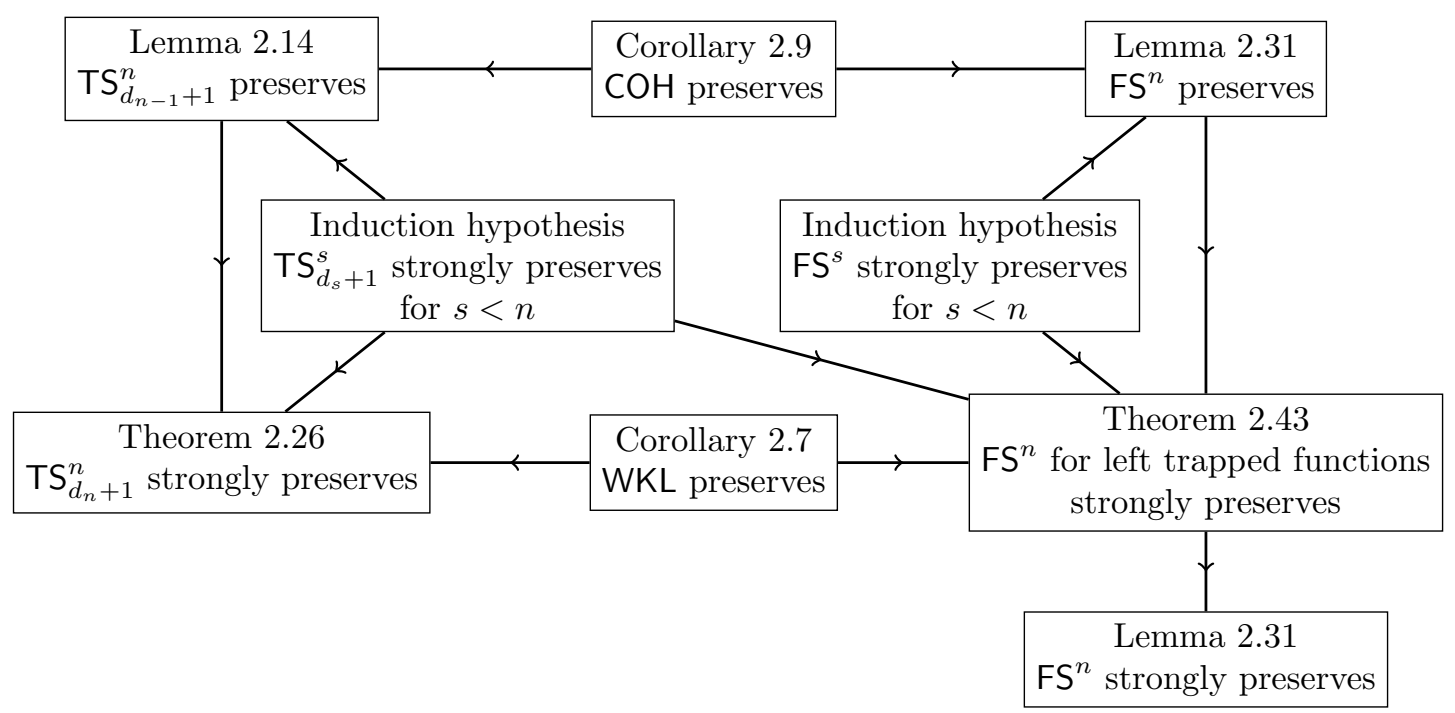

Figure 1. Diagram of dependencies between the proofs of preservation of 2-hyperimmunity. An arrow from $\mathrm{P}$ to $\mathrm{Q}$ means that $\mathrm{Q}$ depends on $\mathrm{P}$.

2.1. Relation with immunity notions. Given a pair of infinite sets $A, B \subseteq \mathbb{N}$, we let $\mathcal{H}(A, B)$ be the bifamily of all finite pairs $(E, F)$ such that $E \subseteq \bar{A}$ and $F \subseteq \bar{B}$. Recall that an infinite set $H$ is $C$-hyperimmune if for every $C$-computable array 11 of finite sets $V_{0}, V_{1}, \ldots$ such that $V_{n}>n$, there is some $n$ such that $V_{n} \subseteq \bar{H}$.

Lemma 2.4. Two sets $A$ and $B$ are $C$-hyperimmune if and only if $\mathcal{H}(A, B)$ is C-2-hyperimmune.

Proof. For convenience, we assume $C=\emptyset$ since the result relativizes. Assume that $A$ and $B$ are hyperimmune, and fix a computable biarray $(\vec{E}, \vec{F})$. By hyperimmunity of $A$ applied to $\vec{E}$, there is some $n$ such that $E_{n} \subseteq \bar{A}$. By hyperimmunity of $B$ applied to $F_{n, 0}, F_{n, 1}, \ldots$, there is some $m$ such that $F_{n, m} \subseteq \bar{B}$. It follows that the biarray $(\vec{E}, \vec{F})$ meets $\mathcal{H}(A, B)$.

Assume now that either $A$ or $B$ is not hyperimmune. Suppose first that $A$ is not hyperimmune. Let $E_{0}, E_{1}, \ldots$ be a computable array of finite sets such that $E_{n}>n$ and $E_{n} \cap A \neq \emptyset$ for every $n$. Then letting $F_{n, m}=\{m+1\}$ for every $m$, the computable biarray $(\vec{E}, \vec{F})$ does not meet $\mathcal{H}(A, B)$. Suppose now that $B$ is not hyperimmune. Let $D_{0}, D_{1}, \ldots$ be a computable array of finite sets such that $D_{n}>n$ and $D_{n} \cap B \neq \emptyset$ for every $n$. Then letting $E_{n}=\{n+1\}$ and $F_{n, m}=D_{m}$ for every $m, n \in \omega$, the computable biarray $(\vec{E}, \vec{F})$ does not meet $\mathcal{H}(A, B)$. In both cases, $\mathcal{H}(A, B)$ is not 2-hyperimmune.

It follows that if a problem $\mathrm{P}$ preserves 2-hyperimmunity, then it also preserves 2 hyperimmunities, in the sense of Definition 6.2.2 in [17. Since SADS is known not to preserve 2 hyperimmunities (see Corollary 10.3.5 in [17]), we can immediatly

\footnotetext{
${ }^{1}$ By a computable array of finite sets $V_{0}, V_{1}, \cdots$, we mean a computable function $\alpha: \omega \rightarrow \omega$ such that $\alpha(n)$ is the canonical index of $V_{n}$.
} 
conclude that SADS does not reserve 2-hyperimmunity. We will nevertheless recall the argument.

\section{Corollary 2.5. SADS does not preserve 2-hyperimmunity.}

Proof. Fix any stable linear order of order type $\omega+\omega^{*}$ with no computable infinite ascending or descending subsequence. Let $A$ and $B$ be the $\omega$ and $\omega^{*}$ part, respectively. By Lemma 41 in [16, $A$ and $B$ are hyperimmune. Therefore, by Lemma 2.4 $\mathcal{H}(A, B)$ is 2-hyperimmune. For any infinite ascending or descending sequence $G$ is a subset of $A$ or $B$, respectively. It follows that either $A$, or $B$ is not $G$-hyperimmune, and by Lemma 2.4. $\mathcal{H}(A, B)$ is not $G$-2-hyperimmune. It follows that SADS does not preserve 2-hyperimmunity.

Whenever a Turing degree contains no $C$-hyperimmune set, it is said to be $C$ hyperimmune-free. A Turing degree $\mathbf{d}$ is known to be $C$-hyperimmune-free if and only if every function bounded by $\mathbf{d}$ is dominated by a $C$-computable function (see Theorem III.3.8 in [12]).

Lemma 2.6. Let $\mathcal{H}$ be a $C$-2-hyperimmune bifamily and $G$ be of $C$-hyperimmunefree degree. Then $\mathcal{H}$ is $C \oplus G$-2-hyperimmune.

Proof. Again, assume $C=\emptyset$ since the result relativizes. Fix a bifamily $\mathcal{H}$, and a set $G$ of hyperimmune-free degree such that $\mathcal{H}$ is not $G$-2-hyperimmune. We want to show that $\mathcal{H}$ is not 2-hyperimmune. Let $(\vec{E}, \vec{F})$ be a $G$-computable biarray which does not meet $\mathcal{H}$. In particular, the function $f$ defined by $f(n, m)=$ $\max E_{n}, F_{n, m}$ is $G$-computable, and is therefore dominated by a computable function $g$. Define the computable biarray $(\vec{K}, \vec{L})$ by $K_{n}=\{n+1, \ldots, g(n, 0)\}$ and $L_{n, m}=\{m+1, \ldots, g(n, m)\}$. It is easy to see that $E_{n} \subseteq K_{n}$ and $F_{n, m} \subseteq L_{n, m}$ for every $n, m \in \omega$. Indeed, $E_{n}>n$ and $\max E_{n} \leq f(n, 0) \leq g(n, 0)$ so $E_{n} \subseteq$ $\{n+1, \ldots, g(n, 0)\}$. Similarly, $F_{n, m}>m$ and $\max F_{n, m} \leq f(n, m) \leq g(n, m)$, so $F_{n, m} \subseteq\{m+1, \ldots, g(n, m)\}$. Since $(\vec{E}, \vec{F})$ does not meet $\mathcal{H},\left(E_{n}, F_{n, m}\right) \notin \mathcal{H}$, and by downward-closure of $\mathcal{H}$ under the subset relation, $\left(K_{n}, L_{n, m}\right) \notin \mathcal{H}$. It follows that $(\vec{K}, \vec{L})$ does not meet $\mathcal{H}$ and therefore that $\mathcal{H}$ is not 2-hyperimmune.

Corollary 2.7. WKL preserves 2-hyperimmunity.

Proof. Let $\mathcal{H}$ be a 2-hyperimmune family, and let $T$ be an infinite computable binary tree. By the hyperimmune-free basis theorem [7, there is an infinite path $P \in[T]$ which is $C$-hyperimmune-free. By Lemma $2.6, \mathcal{H}$ is $P$-2-hyperimmune.

Given a bifamily $\mathcal{H}$, let $\mathcal{B}(\mathcal{H}) \subseteq \omega^{\omega}$ be the closed set of all $X$ such that for every $m, n \in \omega, X(\langle 0, n\rangle)$ and $X(\langle 1, n, m\rangle)$ are finite sets $E_{n}>n$ and $F_{n, m}>m$ such that $\left(E_{n}, F_{n, m}\right) \notin \mathcal{H}$.

Lemma 2.8. A bifamily $\mathcal{H}$ is $C$-2-hyperimmune if and only if $\mathcal{B}(\mathcal{H})$ has no $C$ computable member.

Proof. The members of $\mathcal{B}(\mathcal{H})$ are precisely the biarrays which fail meeting $\mathcal{H}$. The equivalence follows immediatly.

Corollary 2.9. $\mathrm{COH}$ preserves 2-hyperimmunity. 
Proof. Let $\mathcal{H}$ be a 2-hyperimmune family, and let $\vec{R}=R_{0}, R_{1}, \ldots$ be an infinite computable sequence ${ }^{2}$ of sets. By Lemma 2.8, $\mathcal{B}(\mathcal{H})$ has no computable member. By [13, Corollary 2.9], there is an $\vec{R}$-cohesive set $G$ such that $\mathcal{B}(\mathcal{H})$ has no $G$ computable member. By Lemma 2.8. $\mathcal{H}$ is $G$-2-hyperimmune.

2.2. $\mathrm{STS}_{3}^{2}$ and SEM do not preserve 2-hyperimmunity. Before proving that $\mathrm{STS}_{3}^{2}$ and SEM do not preserve 2-hyperimmunity, we must first introduce some notation.

Given a stable coloring $f:[\omega]^{2} \rightarrow 3$ and two sets $E<F$, we write $E \rightarrow_{i} F$ for $(\forall x \in E)(\forall y \in F) f(x, y)=i$. For every $i<3$, we let $C_{i}(f)=\left\{x:\left(\forall^{\infty} y\right) f(x, y)=\right.$ $i\}$. Finally, given a stable coloring $f:[\omega]^{2} \rightarrow 3$, we let $\mathcal{H}(f)$ be the bifamily of all pairs $(E, F)$ such that $E<F, E \subseteq C_{1}(f), F \subseteq C_{2}(f)$, and $E \rightarrow_{0} F$.

Proposition 2.10. There is a stable computable coloring $f:[\omega]^{2} \rightarrow 3$ such that $\mathcal{H}(f)$ is 2-hyperimmune.

Proof. We build the coloring $f:[\omega]^{2} \rightarrow 3$ by a finite injury priority argument. For every $e \in \omega$, we want to satisfy the following requirement:

$$
\begin{aligned}
& \mathcal{R}_{e} \text { :If } \Phi_{e} \text { is total, then there is some } n, m \in \omega \text { such that } \\
& \qquad \Phi_{e}(n ; 1) \subseteq C_{1}(f), \Phi_{e}(n, m ; 2) \subseteq C_{2}(f) \text { and } \Phi_{e}(n ; 1) \rightarrow_{0} \Phi_{e}(n, m ; 2) .
\end{aligned}
$$

The requirements are given the usual priority ordering $\mathcal{R}_{0}<\mathcal{R}_{1}<\ldots$ Initially, the requirements are neither partially, nor fully satisfied.

(i) A requirement $\mathcal{R}_{e}$ requires a first attention at stage $s$ if it is not partially satisfied and $\Phi_{e, s}(n ; 1) \downarrow=E$ for some set $E \subseteq\{e+1, \ldots, s-1\}$ such that no element in $E$ is restrained by a requirement of higher priority. If it receives attention, then it puts a restrain on $E$, commit the elements of $E$ to be in $C_{0}(f)$, and is declared partially satisfied.

(ii) A requirement $\mathcal{R}_{e}$ requires a second attention at stage $s$ if it is not fully satisfied, and $\Phi_{e, s}(n ; 1) \downarrow=E$ and $\Phi_{e, s}(n, m ; 2) \downarrow=F$ for some sets $E, F \subseteq$ $\{e+1, \ldots, s-1\}$ such that $E \rightarrow_{0} F$ and which are not restrained by a requirement of higher priority. If it receives attention, then it puts a restrain on $E \cup F$, commit the elements of $E$ to be in $C_{1}(f)$, the elements of $F$ to be in $C_{2}(f)$, and is declared fully satisfied.

At stage 0 , we let $f=\emptyset$. Suppose that at stage $s$, we have defined $f(x, y)$ for every $x<y<s$. For every $x<s$, if it is committed to be in some $C_{i}(f)$, set $f(x, s)=i$, and otherwise set $f(x, s)=0$. Let $\mathcal{R}_{e}$ be the requirement of highest priority which requires attention. If $\mathcal{R}_{e}$ requires a second attention, then execute the second procedure, otherwise execute the first one. In any case, reset all the requirements of lower priorities by setting them unsatisfied, releasing all their restrains, and go to the next stage. This completes the construction. On easily sees by induction that each requirement acts finitely often, and is eventually fully satisfied. This procedure also yields a stable coloring.

Corollary 2.11. $\mathrm{STS}_{3}^{2}$ does not preserve 2-hyperimmunity.

Proof. Let $f$ be the stable computable coloring of Proposition 2.10. Let $G=\left\{x_{0}<\right.$ $\left.x_{1}<\ldots\right\}$ be an infinite $f$-thin set. We claim that $\mathcal{H}(f)$ is not $G$-2-hyperimmune. Indeed, let $(\vec{E}, \vec{F})$ be the $G$-computable biarray defined by $E_{n}=\left\{x_{n}\right\}$ and $F_{n, m}=$

${ }^{2} \mathrm{By}$ a computable sequence of sets $R_{0}, R_{1}, \cdots$ we mean $R_{n}$ is uniformly computable. 
THE REVERSE MATHEMATICS OF THE THIN SET AND THE ERDŐS-MOSER THEOREMS9

$\left\{x_{n+m}\right\}$. Fix any $n$. Suppose that $E_{n} \subseteq C_{1}(f)$ and $F_{n, m} \subseteq C_{2}(f)$ (if such $E_{n}, F_{n, m}$ does not exist then we are done). In other words, for every sufficiently large $k$, $f\left(x_{n}, x_{k}\right)=1$ and $f\left(x_{n+m}, x_{k}\right)=2$. It follows that $G$ must be $f$-thin for color 0 3 therefore $E_{n} t_{0} F_{n, m}$ and so $\left(E_{n}, F_{n, m}\right) \notin \mathcal{H}(f)$. The $G$-computable biarray $(\vec{E}, \vec{F})$ does not meet $\mathcal{H}(f)$, so $\mathcal{H}(f)$ is not $G$-2-hyperimmune.

Corollary 2.12. SEM does not preserve 2-hyperimmunity.

Proof. Let $f:[\omega]^{2} \rightarrow 3$ be the stable computable coloring of Proposition 2.10 Let $T$ be the stable computable tournament defined for every $x<y$ by $T(x, y)$ iff $f(x, y)=1$. Let $G=\left\{x_{0}<x_{1}<\ldots\right\}$ be an infinite transitive subtournament. We claim that $\mathcal{H}(f)$ is not $G$-2-hyperimmune. Indeed, let $(\vec{E}, \vec{F})$ be the $G$-computable biarray defined by $E_{n}=\left\{x_{n}\right\}$ and $F_{n, m}=\left\{x_{n+m}\right\}$. Fix $n$. Suppose that $E_{n} \subseteq$ $C_{1}(f)$ and $F_{n, m} \subseteq C_{2}(f)$ (if such $E_{n}, F_{n, m}$ does not exist then we are done). In other words, for every sufficiently large $k, f\left(x_{n}, x_{k}\right)=1$ and $f\left(x_{n+m}, x_{k}\right)=$ 2 , so $T\left(x_{n}, x_{k}\right)$ and $T\left(x_{k}, x_{n+m}\right)$ will hold. By transitivity of $G, T\left(x_{n}, x_{n+m}\right)$ must hold, so $f\left(x_{n}, x_{n+m}\right)=1$. It follows that $E_{n} t_{0} F_{n, m}$ and so $\left(E_{n}, F_{n, m}\right) \notin$ $\mathcal{H}(f)$. The $G$-computable biarray $(\vec{E}, \vec{F})$ does not meet $\mathcal{H}(f)$, so $\mathcal{H}(f)$ is not $G-2-$ hyperimmune.

2.3. $\mathrm{TS}_{4}^{2}$ preserves 2-hyperimmunity. The purpose of this section is to prove the following theorem.

Theorem 2.13. $\mathrm{TS}_{4}^{2}$ preserves 2-hyperimmunity.

This will be generalized to arbitrary tuples in the next section. The notion of preservation of 2-hyperimmunity for $\mathrm{TS}_{k}^{n+1}$ relates to the notion of strong preservation of 2-hyperimmunity for $\mathrm{TS}_{k}^{n}$ in the following sense.

Lemma 2.14. Fix some $n \geq 1$ and $k \geq 2$. If $\mathrm{TS}_{k}^{n}$ strongly preserves 2-hyperimmunity, then $\mathrm{TS}_{k}^{n+1}$ preserves 2-hyperimmunity.

Proof. Let $\mathcal{H}$ be a 2-hyperimmune family, and $f:[\omega]^{n+1} \rightarrow k$ be a computable instance of $\mathrm{TS}_{k}^{n+1}$. Let $\vec{R}=\left\langle R_{\sigma, i}: \sigma \in[\omega]^{n}, i<k\right\rangle$ be the computable family of sets defined for every $\sigma \in[\omega]^{n}$ and $i<k$ by

$$
R_{\sigma, i}=\{x \in \omega: f(\sigma, x)=i\}
$$

Since $\mathrm{COH}$ preserves 2-hyperimmunity (Corollary 2.9 , there is an $\vec{R}$-cohesive set $G$ such that $\mathcal{H}$ is $G$-2-hyperimmune. Let $g:[\omega]^{n} \rightarrow k$ be the $\Delta_{2}^{0, G}$ instance of $\mathrm{TS}_{k}^{n}$ defined for every $\sigma \in[\omega]^{n}$ by

$$
g(\sigma)=\lim _{x \in G} f(\sigma, x)
$$

By strong preservation of $\mathrm{TS}_{k}^{n}$, there is an infinite $g$-thin set $H$ such that $\mathcal{H}$ is $G \oplus H$-2-hyperimmune. By thinning out the set $H$, we obtain an infinite $G \oplus H$ computable $f$-thin set $\tilde{H}$. In particular, $\mathcal{H}$ is $\tilde{H}$-2-hyperimmune.

It therefore remains to prove the following theorem.

Theorem 2.15. $\mathrm{TS}_{4}^{1}$ strongly preserves 2-hyperimmunity.

$\overline{{ }^{3} \text { Given } f:[\omega]^{n}} \rightarrow \omega$, a set $G$ is $f$-thin for color $i$ iff $i \notin f[G]^{n}$. 
Proof. For notation convenience, we prove the non-relativized version. Let $\mathcal{H}$ be a 2-hyperimmune bifamily, and let $f: \omega \rightarrow 4$ be an arbitrary instance of $\mathrm{TS}_{4}^{1}$. Without loss of generality, assume that there is no infinite subset $H$ of $f^{-1}(i)$ such that $\mathcal{H}$ is $H$-2-hyperimmune (as otherwise we are done). We are going to

build 4 infinite sets $\left(G_{i}: i<4\right)$ such that $G_{i}$ is $f$-thin for color $i$ and

$\mathcal{H}$ is $G_{i}$-2-hyperimmune for some $i<4$.

We are going to build the sets $G_{i}$ by a Mathias forcing whose conditions are tuples $\left(F_{0}, F_{1}, F_{2}, F_{3}, X\right)$, where $\left(F_{i}, X\right)$ is a Mathias condition, $F_{i}$ is $f$-thin for color $i$ and $\mathcal{H}$ is $X$-2-hyperimmune. A condition $d=\left(E_{0}, E_{1}, E_{2}, E_{3}, Y\right)$ extends $c=$ $\left(F_{0}, F_{1}, F_{2}, F_{3}, X\right)$ (written $\left.d \leq c\right)$ if $\left(E_{i}, Y\right)$ Mathias extends $\left(F_{i}, X\right)$ for every $i<4$.

The first lemma ensures that every sufficiently generic filter for this notion of forcing will induce four infinite sets.

Lemma 2.16. For every condition $c=\left(F_{0}, F_{1}, F_{2}, F_{3}, X\right)$ and every $i<4$, there is an extension $d=\left(E_{0}, E_{1}, E_{2}, E_{3}, Y\right)$ of $c$ such that $\left|E_{i}\right|>\left|F_{i}\right|$.

Proof. Fix $c$ and $i<4$. Note that $X \backslash f^{-1}(i)$ is infinite, since otherwise it contradicts with our assumption that $\mathcal{H}$ is $X$-2-hyperimmune. Let $x \in X \backslash f^{-1}(i)$ with $x>F_{i}$. The condition $d=\left(E_{0}, E_{1}, E_{2}, E_{3}, X\right)$ defined by $E_{i}=F_{i} \cup\{x\}$, and $E_{j}=F_{j}$ for $j \neq i$ is the desired extension of $c$.

A 4 -tuple of sets $G_{0}, G_{1}, G_{2}, G_{3}$ satisfies a condition $c=\left(F_{0}, F_{1}, F_{2}, F_{3}, X\right)$ if $G_{i}$ satisfies the Mathias condition $\left(F_{i}, X\right)$ for every $i<4$. A condition $c$ forces a formula $\varphi\left(G_{0}, G_{1}, G_{2}, G_{3}\right)$ if the formula holds for every 4-tuple of sets $G_{0}, G_{1}, G_{2}, G_{3}$ satisfying $c$. Given any $e_{0}, e_{1}, e_{2}, e_{3}$, we want to satisfy the following requirements:

$$
\mathcal{R}_{e_{0}, e_{1}, e_{2}, e_{3}}: \mathcal{R}_{e_{0}}^{0} \vee \mathcal{R}_{e_{1}}^{1} \vee \mathcal{R}_{e_{2}}^{2} \vee \mathcal{R}_{e_{3}}^{3}
$$

where $\mathcal{R}_{e}^{i}$ is the requirement:

$$
\text { If } \Phi_{e}^{G_{i}} \text { is total, then it meets } \mathcal{H} \text {. }
$$

Lemma 2.17. For every condition c and every 4 -tuple of indices $e_{0}, e_{1}, e_{2}, e_{3}$, there is an extension $d$ of $c$ forcing $\mathcal{R}_{e_{0}, e_{1}, e_{2}, e_{3}}$.

Lemma 2.17, 2.29, 2.38 and 2.46 are main technical lemmas of Theorem 1.6 (where we prove a condition can be extended to force that a given Turing functional meets $\mathcal{H}$ ). We briefly explain one of the idea of these lemmas (which also appears in the main lemma 3.9 of Theorem 3.6 — a generalization of Seetapun forcing to build weak solution. Usually, given an arbitrary instance $f$ (of a problem), we want to build a solution $G$ to $f$ so that $\Phi^{G}$ has a desired behaviour. Since $f$ is not computable, we cannot search computably among initial segments $F$ of solutions to $f$ (call such $F$ finite solution to $f$ ) such that $\Phi^{F}$ has that behaviour. The idea of Seetapun forcing is to find sufficiently many $F$ so that $\Phi^{F}$ has that behaviour. By "sufficiently many", it means whatever $f$ looks like, one of $F$ is, at least, a finite solution to $f$.

In our case, this means we want to find sufficiently many $F$ such that $\Phi^{F}(n ; 1) \downarrow$ and $\Phi^{F}(n, m ; 2) \downarrow$. But this is not quite enough. It only gives (by compactness) two sets $U_{n, m}, V_{n, m}$ so that for every $g$, there is a finite solution $F$ of $g$ such that $\Phi^{F}(n ; 1) \subseteq U_{n, m} \wedge \Phi^{F}(n, m ; 2) \subseteq V_{n, m}$. i.e., $U_{n, m}$ depends on $m$. So one may want to try this: firstly, search for a sufficient collection $\mathcal{F}$, so that $\Phi^{F}(n ; 1) \downarrow$ for each 
$F \in \mathcal{F}$; then search for a sufficient collection $\mathcal{E}$ each $E \in \mathcal{E}$ extends a member of $\mathcal{F}$ and $\Phi^{E}(n, m ; 2) \downarrow$. Let's see what sufficiency notion should $\mathcal{F}$ satisfy. When $\mathcal{F}$ exists while $\mathcal{E}$ does not, we have that for some instance $g$,

there is no finite solution $E$ of $g$ such that $E$ extends a member of $\mathcal{F}$ and $\Phi^{E}(n, m ; 2) \downarrow$.

We need to find an appropriate $F \in \mathcal{F}$ such that $F$ is a finite solution to $g$ and restrict $G$ so that $G$ extends $F$ and $G$ is a solution to $g$ (because by $(2.2)$, for such $\left.G, \Phi^{G}(n, m ; 2) \uparrow\right)$. By appropriate, it means, at least, $F$ is a finite solution to $f$. The sufficiency notion of $\mathcal{F}$ should guarantee the existence of $F$. This gives the following sufficiency notion: for every two instances $g, h$, there is an $F \in \mathcal{F}$ such that $F$ is a finite solution to both $g, h$. This is exactly the sufficiency notion we use in Lemma 2.17. For more complex problems, $F$ being a finite solution to $f$ is not enough, but it has to ensure that imposing the restriction of $F$ does not cut the candidate space too much. This concern gives rise to more complex sufficiency notion (Definition 2.24, 2.41 and Lemma 2.42, 2.25).

Proof. Fix $c=\left(F_{0}, F_{1}, F_{2}, F_{3}, X\right)$. For notation convenience, we assume $X=\omega$ and $F_{i}=\emptyset$. We define a partial computable biarray as follows. To obtain a desired extension of $c$, we take advantage of the failure of this biarray to meet $\mathcal{H}$ or its non-totality.

Defining $U_{n}$. Given $n \in \omega$, search computably for some finite set $U_{n}>n \|^{7}$ (if it exists) such that for every pair of colorings $g, h: \omega \rightarrow 4$, there are two colors $i_{0}<i_{1}<4$ and two sets $E_{i_{0}}$ and $E_{i_{1}}$ such that for every $i \in\left\{i_{0}, i_{1}\right\}, E_{i}$ is both $g$-thin, $h$-thin for color $i$ and 5

$$
\Phi_{e_{i}}^{E_{i}}(n ; 1) \downarrow \subseteq U_{n} .
$$

Defining $V_{n, m}$. Given $n, m \in \omega$, search computably for some finite set $V_{n, m}>m$ (if it exists) such that for every coloring $g: \omega \rightarrow 4$, there is an $i<4$ and a finite set $E_{i} g$-thin for color $i$ such that

$$
\Phi_{e_{i}}^{E_{i}}(n ; 1) \downarrow \subseteq U_{n} \wedge \Phi_{e_{i}}^{E_{i}}(n, m ; 2) \downarrow \subseteq V_{n, m} .
$$

We now have multiple outcomes, depending on which of $U_{n}$ and $V_{n, m}$ is found.

- Case 1: $U_{n}$ is not found for some $n \in \omega$. By compactness, the following $\Pi_{1}^{0}$ class $\mathcal{P}$ of pairs of colorings $g, h: \omega \rightarrow 4$ is nonempty: there are three indices $i_{0}<i_{1}<i_{2}<4$ such that for every $i \in\left\{i_{0}, i_{1}, i_{2}\right\}$ and every finite set $E_{i}$ being both $g$-thin and $h$-thin for color $i$, we have $\Phi_{e_{i}}^{E_{i}}(n ; 1) \uparrow$.

As WKL preserves 2-hyperimmunity (Corollary 2.7), there is a member $(g, h)$ of $\mathcal{P}$ such that $\mathcal{H}$ is $g \oplus h$-2-hyperimmune. In particular, there are some $i_{0}<i_{1}<i_{2}<4$ such that for every $i \in\left\{i_{0}, i_{1}, i_{2}\right\}$ and every finite $E_{i}$ being $g$-thin, $h$-thin for color $i$, we have $\Phi_{e_{i}}^{E_{i}}(n ; 1) \uparrow$. There must be an

\footnotetext{
${ }^{4}$ More concretely, we mean search the canonical index of $U_{n}$.

${ }^{5} \mathrm{By}$ compactness, if $U_{n}$ is found, there is a sufficient sequence $\left(\mathcal{F}_{i}: i<4\right)$ of finite collections of finite sets so that for every $E \in \mathcal{F}, \Phi_{e_{i}}^{E}(n ; 1) \downarrow \subseteq U_{n}$. Here sufficient means for every two colorings $g, h: \omega \rightarrow 4$, there are $i_{0}<i_{1}<4$ and $E_{i} \in \mathcal{F}_{i}$ for each $i \in\left\{i_{0}, i_{1}\right\}$ so that $E_{i}$ is $g$-thin, $h$-thin for color $i$.
} 
$i \in\left\{i_{0}, i_{1}, i_{2}\right\}$ such that the set $Y=\{x: g(x) \neq i, h(x) \neq i\}$ is infinite ${ }^{6}$ Then clearly $\left(F_{0}, F_{1}, F_{2}, F_{3}, Y\right)$ is an extension of $c$. For every $G$ satisfying $\left(F_{i}, Y\right), G$ is $g$-thin for color $i$. Thus $\Phi_{e_{i}}^{G}(n ; 1) \uparrow$. i.e., $d$ forces $\Phi_{e_{i}}^{G}(n ; 1) \uparrow$, hence $\mathcal{R}_{e_{0}, e_{1}, e_{2}, e_{3}}$.

- Case 2: $U_{n}$ is found, but not $V_{n, m}$ for some $n, m \in \omega$. By compactness, the following $\Pi_{1}^{0}$ class $\mathcal{P}$ of colorings $g: \omega \rightarrow 4$ is nonempty: for every $i<4$ and every finite set $E_{i} g$-thin for color $i$,

$$
\Phi_{e_{i}}^{E_{i}}(n ; 1) \downarrow \subseteq U_{n} \Rightarrow \Phi_{e_{i}}^{E_{i}}(n, m ; 2) \uparrow .
$$

As WKL preserves 2-hyperimmunity (Corollary 2.7), there is a member $g$ of $\mathcal{P}$ such that $\mathcal{H}$ is $g$-2-hyperimmune. By definition of $U_{n}$ (where we take $h=f$ in the definition of $U_{n}$ ), there are some $i_{0}<i_{1}<4$ and some finite sets $E_{i_{0}}$ and $E_{i_{1}}$ such that for every $i \in\left\{i_{0}, i_{1}\right\}, E_{i}$ is both $g$-thin, $f$-thin for color $i$ and

$$
\Phi_{e_{i}}^{E_{i}}(n ; 1) \downarrow \subseteq U_{n} .
$$

In particular, there must be some $i \in\left\{i_{0}, i_{1}\right\}$ such that the set $Y=\{x$ : $g(x) \neq i\}$ is infinite. Consider the extension $d=\left(D_{0}, D_{1}, D_{2}, D_{3}, Y\right)$ of $c$ defined by $D_{i}=F_{i} \cup E_{i}$ and $D_{j}=F_{j}$ for each $j \neq i$. To see $d$ forces $\Phi_{e_{i}}^{G_{i}}(n, m ; 2) \uparrow$ (hence forces $\left.\mathcal{R}_{e_{0}, e_{1}, e_{2}, e_{3}}\right)$, note that for every $G$ satisfying $\left(D_{i}, Y\right), G$ is $g$-thin for color $i$. But $\Phi_{e_{i}}^{D_{i}}(n ; 1) \downarrow \subseteq U_{n}$. Thus, by definition of $g$ (namely 2.3), $\Phi_{e_{i}}^{G}(n, m ; 2) \uparrow$.

- Case 3: $U_{n}$ and $V_{n, m}$ are found for every $n, m \in \omega$. By 2-hyperimmunity of $\mathcal{H}$, there is some $n, m \in \omega$ such that $\left(U_{n}, V_{n, m}\right) \in \mathcal{H}$. In particular, by definition of $V_{n, m}$ (where we take $g=f$ in the definition of $V_{n, m}$ ), there is some $i<4$ and some finite set $E_{i}$ such that $E_{i}$ is $f$-thin for color $i$ and

$$
\Phi_{e_{i}}^{E_{i}}(n ; 1) \downarrow \subseteq U_{n} \wedge \Phi_{e_{i}}^{E_{i}}(n, m ; 2) \downarrow \subseteq V_{n, m} .
$$

The condition $\left(D_{0}, D_{1}, D_{2}, D_{3}, X\right)$ defined by $D_{i}=F_{i} \cup E_{i}$ and $D_{j}=F_{j}$ for each $j \neq i$ is an extension of $c$ forcing $\mathcal{R}_{e_{0}, e_{1}, e_{2}, e_{3}}$.

This completes the proof of Lemma 2.17

Let $\mathcal{F}=\left\{c_{0}, c_{1}, \ldots\right\}$ be a sufficiently generic filter for this notion of forcing, where $c_{s}=\left(F_{0, s}, F_{1, s}, F_{2, s}, F_{3, s}, X_{s}\right)$, and let $G_{i}=\bigcup_{s} F_{i, s}$. By definition of a condition, for every $i<4, G_{i}$ is $f$-thin for color $i$. By Lemma 2.16, $G_{i}$ are all infinite, and by Lemma 2.17, there is some $i<4$ such that $\mathcal{H}$ is $G_{i}$-2-hyperimmune. This completes the proof of Theorem 2.15 .

For $\mathrm{TS}_{3}^{2}$, its relation with $\mathrm{EM}$ is unclear.

Question 2.18. Does $\mathrm{TS}_{3}^{2}$ implies EM?

${ }^{6}$ This is where the argument works with $\mathrm{TS}_{4}^{1}$ and fails with $\mathrm{TS}_{3}^{1}$ : with $\mathrm{TS}_{3}^{1}$, we would only get two colors $\left\{i_{0}, i_{1}\right\}$, and if $g$ and $h$ are the constant functions $i_{0}$ and $i_{1}$, respectively, the set $Y$ is finite for every $i \in\left\{i_{0}, i_{1}\right\}$. 
THE REVERSE MATHEMATICS OF THE THIN SET AND THE ERDŐS-MOSER THEOREMG3

2.4. Generalized cohesiveness preserves 2-hyperimmunity. In order to prove that $\mathrm{TS}_{k}^{n}$ and $\mathrm{FS}^{n}$ preserve 2-hyperimmunity for sufficiently large $k$, we first need to prove the following technical theorem, which thin out colors while preserving 2-hyperimmunity. The proof is a slight adaptation of 13 to 2-hyperimmunity. We however reprove it for the sake of completeness. We will need the case $t=n-1$ for $\mathrm{TS}_{k}^{n}$, and the case $t=n$ for $\mathrm{FS}^{n}$. Fix a set $C$, a bifamily $\mathcal{H}$ which is $C-2$ hyperimmune, an infinite set $X \leq_{T} C$; let $f:[\omega]^{n} \rightarrow k$ be a coloring.

Proposition 2.19. Assume $\mathrm{TS}_{d_{s}+1}^{s}$ strongly preserves 2-hyperimmunity for each $0<s<n$. Then there exists an infinite set $G \subseteq X$ such that $\mathcal{H}$ is $C \oplus G$ 2-hyperimmune, and for every $\sigma \in[\omega]<\omega$ such that $0<|\sigma|<n$, there is a set $I_{\sigma} \subseteq\{0, \ldots, k-1\}$ such that $\left|I_{\sigma}\right| \leq d_{n-|\sigma|}$ and

$$
(\exists b)\left(\forall \tau \in[G \cap(b,+\infty)]^{n-|\sigma|}\right) f(\sigma, \tau) \in I_{\sigma} .
$$

Proof. For notation convenience, assume $X=\omega$ and $C=\emptyset$. Our forcing conditions are Mathias conditions $(F, Y)$ where $\mathcal{H}$ is $Y$-2-hyperimmune. The first lemma shows that $\mathcal{H}$ will be $G$-2-hyperimmune for every sufficiently generic filter. Given $e$, let $\mathcal{R}_{e}$ be the requirement:

$$
\text { If } \Phi_{e}^{G} \text { is total, then it meets } \mathcal{H} \text {. }
$$

Lemma 2.20. Given a condition $c=(F, Y)$ and an index $e \in \omega$, there is an extension $d$ forcing $\mathcal{R}_{e}$.

Proof. This simply follows by finite extension argument. Again for notation convenience, assume $F=\emptyset$ and $Y=\omega$. We define a partial computable biarray as follows.

Defining $U_{n}$. Given $n \in \omega$, search computably for some finite set $U_{n}>n$ (if it exists) and a finite set $E$ such that

$$
\Phi_{e}^{E}(n ; 1) \downarrow=U_{n} .
$$

Defining $V_{n, m}$. Given $n, m \in \omega$, search computably for some finite set $V_{n, m}>m$ (if it exists) and a finite set $E$ such that

$$
\Phi_{e}^{E}(n ; 1) \downarrow=U_{n} \wedge \Phi_{e}^{E}(n, m ; 2) \downarrow=V_{n, m} .
$$

We now have multiple outcomes, depending on which $U_{n}$ and $V_{n, m}$ is found.

- Case 1: $U_{n}$ is not found for some $n \in \omega$. Then the condition $c=(F, Y)$ already forces $\Phi_{e}^{G}(n ; 1) \uparrow$ and therefore forces $\mathcal{R}_{e}$.

- Case 2: $U_{n}$ is found, but not $V_{n, m}$ for some $n, m \in \omega$. By definition of $U_{n}$, there is a finite set $E$ such that

$$
\Phi_{e}^{E}(n ; 1) \downarrow=U_{n}
$$

The condition $d=(E, Y)$ is an extension of $c$ forcing $\Phi_{e}^{G}(n, m ; 2) \uparrow$.

- Case 3: $U_{n}$ and $V_{n, m}$ are found for every $n, m \in \omega$. By 2-hyperimmunity of $\mathcal{H}$, there is some $n, m \in \omega$ such that $\left(U_{n}, V_{n, m}\right) \in \mathcal{H}$. In particular, by definition of $V_{n, m}$, there is a finite set $E$ such that

$$
\Phi_{e}^{E}(n ; 1) \downarrow=U_{n} \wedge \Phi_{e}^{E}(n, m ; 2) \downarrow=V_{n, m}
$$

The condition $d=(E, Y)$ is an extension of $c$ forcing $\Phi_{e}^{G}$ to meet $\mathcal{H}$, and therefore forcing $\mathcal{R}_{e}$. 
This completes the proof of Lemma 2.20 .

Lemma 2.21. For every condition $(F, Y)$ and $\sigma \in[\omega]<\omega$ such that $0<|\sigma|<n$, there is a finite set $I \subseteq\{0, \ldots, k-1\}$ with $|I| \leq d_{n-|\sigma|}$ and an extension $(F, \tilde{Y})$ such that

$$
\left(\forall \tau \in[\tilde{X}]^{n-|\sigma|}\right) f(\sigma, \tau) \in I .
$$

Proof. This simply follows by strong preservation of $\mathrm{TS}_{d_{n-|\sigma|}}^{n-|\sigma|}$. Define the function $g:[Y]^{n-|\sigma|} \rightarrow k$ by $g(\tau)=f(\sigma, \tau)$. Since $\mathrm{TS}_{d_{n-|\sigma|}-|\sigma|}^{n-\mid}$ strongly preserves 2-hyperimmunity (since $0<n-|\sigma|<n$ ), there exists an infinite $\tilde{Y} \subseteq Y$ and a finite set $I \subseteq\{0, \ldots, k-1\}$ such that $\mathcal{H}$ is $\tilde{Y}$-2-hyperimmune, $|I| \leq \bar{d}_{n-|\sigma|}$, and $\left(\forall \tau \in[\tilde{Y}]^{n-|\sigma|}\right) f(\sigma, \tau) \in I$. The condition $(F, \tilde{Y})$ is the desired extension.

Let $\mathcal{F}=\left\{c_{0}, c_{1}, \ldots\right\}$ be a sufficiently generic filter containing $(\emptyset, \omega)$, where $c_{s}=$ $\left(F_{s}, X_{s}\right)$. The filter $\mathcal{F}$ yields a unique infinite set $G=\bigcup_{s} F_{s}$. By Lemma 2.20, $\mathcal{H}$ is $G$-2-hyperimmune. By Lemma 2.21, $G$ satisfies the property of the theorem. This completes the proof of Proposition 2.19

2.5. $\mathrm{TS}^{n}$ preserves 2-hyperimmunity. Define the sequence $d_{0}, d_{1}, \ldots$ by induction as follows:

$$
d_{0}=1 \quad d_{n}=2 d_{n-1}+\sum_{0 \leq s<n} d_{s-1} d_{n-s-1}+\sum_{0<s<n} d_{s} d_{n-s} \quad \text { for } n>1
$$

The purpose of this section is to prove the following theorem.

Theorem 2.22. $\mathrm{TS}_{d_{n}+1}^{n}$ strongly preserves 2-hyperimmunity for every $n \geq 1$.

Proof. We prove by induction over $n \geq 1$ that $\mathrm{TS}_{d_{n-1}+1}^{n}$ preserves 2-hyperimmunity, and that $\mathrm{TS}_{d_{n}+1}^{n}$ strongly preserves 2-hyperimmunity. If $n=1, \mathrm{TS}_{2}^{1}$ is a computably true statement, that is, every instance has a solution computable in the instance, so $\mathrm{TS}_{2}^{1}$ preserves 2-hyperimmunity. On the other hand, $\mathrm{TS}_{4}^{1}$ strongly preserves 2hyperimmunity follow from Theorem 2.15 . If $n>1$, then by induction hypothesis, $\mathrm{TS}_{d_{n-1}+1}^{n-1}$ strongly preserves 2-hyperimmunity, so by Lemma 2.14 . $\mathrm{TS}_{d_{n-1}+1}^{n}$ preserves 2-hyperimmunity. Assuming by induction hypothesis that $\mathrm{TS}_{d_{s}+1}^{s}$ strongly preserves 2-hyperimmunity for every $0<s<n$, and that $\mathrm{TS}_{d_{n-1}+1}^{n}$ preserves 2hyperimmunity, by Theorem 2.26 . $\mathrm{TS}_{d_{n}+1}^{n}$ strongly preserves 2-hyperimmunity.

We need to prove Theorem 2.26 to complete the proof of Theorem 2.22 . We start with the following technical lemma which thin out colors while preserve 2hyperimmune. Fix a set $C$, a bifamily $\mathcal{H}$ which is $C$-2-hyperimmune, an infinite set $X \leq_{T} C$ and a coloring $f:[\omega]^{n} \rightarrow k$.

Lemma 2.23. Assume $\mathrm{TS}_{d_{s}+1}^{s}$ strongly preserves 2-hyperimmunity for every $0<$ $s<n$. Then there is an infinite set $Y \subseteq X$ so that $\mathcal{H}$ is $C \oplus Y$-2-hyperimmune, and a finite set $I \subseteq\{0, \ldots, k-1\}$ with $|I| \leq \sum_{0<s<n} d_{s} d_{n-s}$ such that for each $0<s<n$,

$$
\left(\forall \sigma \in[Y]^{s}\right)(\exists b)\left(\forall \tau \in[Y \cap(b, \infty)]^{n-s}\right) f(\sigma, \tau) \in I
$$

Proof. For notation convenience, assume $C=\emptyset$. Apply Proposition 2.19 to get an infinite set $X_{0} \subseteq X$ so that $\mathcal{H}$ is $X_{0}$-2-hyperimmune and for every $\sigma \in[\omega]<\omega$ with $0<|\sigma|<n$, there is an $I_{\sigma}$ such that $\left|I_{\sigma}\right| \leq d_{n-|\sigma|}$ and

$$
(\exists b)\left(\forall \tau \in\left[X_{0} \cap(b,+\infty)\right]^{n-|\sigma|}\right) f(\sigma, \tau) \in I_{\sigma} .
$$


THE REVERSE MATHEMATICS OF THE THIN SET AND THE ERDŐS-MOSER THEOREMB5

For each $0<s<n$ and $\sigma \in[\omega]^{s}$, let $F_{s}(\sigma)=I_{\sigma}$. Since $\mathrm{TS}_{d_{s}+1}^{s}$ strongly preserves 2-hyperimmunity, for each $0<s<n$, there is an infinite set $Y \subseteq X_{0}$ such that $\mathcal{H}$ is $Y$-2-hyperimmune and such that $\left|F_{s}[Y]^{s}\right| \leq d_{s}$ for all $0<s<n$. Let $\mathcal{I}_{s}=F_{s}[Y]^{s}$ for each $0<s<n$, and let $I=\bigcup_{J \in \mathcal{I}_{s}, 0<s<n} J$. Then

$$
|I| \leq \sum_{0<s<n} d_{s} d_{n-s}
$$

We now check that the property is satisfied. Fix an $0<s<n$, a $\sigma \in[Y]^{s}$ and let $b \in \omega$ be sufficiently large. Because $Y \subseteq X_{0}$,

$$
\left(\forall \tau \in[Y \cap(b,+\infty)]^{n-s}\right) f(\sigma, \tau) \in I_{\sigma} .
$$

So $F_{s}(\sigma)=I_{\sigma}$, but $\sigma \in[Y]^{s}$, hence $I_{\sigma} \in \mathcal{I}_{s}$. It follows that

$$
\left(\forall \tau \in[Y \cap(b,+\infty)]^{n-s}\right) f(\sigma, \tau) \in I .
$$

This completes the proof.

We need to prove a second lemma saying that if we have sufficiently many finite thin sets, one of them is extendible into an infinite one. This argument is a generalization of case 2 of Lemma 2.17 .

Definition 2.24 (TS-sufficient). Let $\left(\mathcal{F}_{i}: i<p\right)$ be a $p$-tuple of finite collections of finite sets. We say $\left(\mathcal{F}_{i}: i<p\right)$ is $n$-TS-sufficient iff for every sequence of colorings $\left(f_{s, j}:[\omega]^{s} \rightarrow d_{n}+1\right)_{0 \leq s<n, j<d_{n-s-1}}$, there is an $i<p$, an $F \in \mathcal{F}_{i}$ such that $F$ is $f_{s, j}$-thin for color $i$ for all $0 \leq s<n, j<d_{n-s-1}$.

In our application, $p$ will be smaller than $d_{n}$. Let $\left(\mathcal{F}_{i}: i<p\right)$ be a $p$-tuple of finite collections of finite sets.

Lemma 2.25. Assume $\mathrm{TS}_{d_{s}+1}^{s}$ strongly preserves 2-hyperimmunity for every $0<$ $s<n$. Suppose $f \leq_{T} C,\left(\mathcal{F}_{i}: i<p\right)$ is $n$-TS-sufficient and for every $i<p, \mathcal{F}_{i}$ is $f$-thin for color $\sqrt{7}$. Then there exists an $i<p$, an $F \in \mathcal{F}_{i}$ and an infinite set $Y \subseteq X$ such that $F \cup Y$ is $f$-thin for color $i$ and $\mathcal{H}$ is $Y \oplus C$-2-hyperimmune.

Proof. Again, for notation convenience, assume $C=\emptyset$. Let $E=\cup_{F \in \mathcal{F}_{i}, i<p} F$. For every $s<n$, every $\sigma \in[E]^{s}$, let coloring $f_{\sigma}:[\omega]^{n-s} \rightarrow d_{n}+1$ be defined as $f_{\sigma}(\tau)=f(\sigma, \tau)$. By Lemma 2.14. $\mathrm{TS}_{d_{s-1}+1}^{s}$ admits preservation of 2-hyperimmunity for $0 \leq s \leq n$ ( set $d_{-1}=1$ ), so there is an infinite set $Y \subseteq X$ with $\mathcal{H}$ being $Y$ 2-hyperimmune such that for every $0 \leq s<n$, every $\sigma \in[E]^{s}$, there is a $I_{\sigma}$ with $\left|I_{\sigma}\right| \leq d_{n-s-1}$ such that

$$
f_{\sigma}[Y]^{n-s} \subseteq I_{\sigma} .
$$

For every $0 \leq s<n$ and $j<d_{n-s-1}$, let $f_{s, j}$ be the coloring on $[E]^{s}$ such that $f_{s, j}(\sigma)$ is the $j$ th element of $I_{\sigma}$.

By $n$-TS-sufficient of $\left(\mathcal{F}_{i}: i<p\right)$, there is a $i<p$, an $F \in \mathcal{F}_{i}$ such that $F$ is $f_{s, j}$-thin for color $i$ for all $0 \leq s<n$ and $j<d_{n-s-1}$. In particular, $i \notin I_{\emptyset}$ since $F$ is $f_{0, j}$-thin for color $i$ (and $f_{0, j} \equiv$ the $j$ th element of $I_{\emptyset}$ ). This means $Y$ is $f$-thin for color $i$.

We show that $F \cup Y$ is $f$-thin for color $i$. To see this, let $\sigma \in[F]^{<\omega}, \tau \in[Y]^{<\omega}$ with $|\sigma \cup \tau|=n$. When $|\sigma|=n$ or $|\tau|=n, f(\sigma, \tau) \neq i$ follows from $f$-thin for color $i$ of $F$ and $Y$ respectively. When $|\sigma|=s$ with $0<s<n$, since $F$ is $f_{s, j}$-thin

${ }^{7}$ Each member of $\mathcal{F}_{i}$ is $f$-thin for color $i$. 
for color $i$ for all $j<d_{n-s-1}$, we have $f_{s, j}(\sigma) \neq i$ for all $j<d_{n-s-1}$. This means $i \notin I_{\sigma}$. Thus $f(\sigma, \tau) \neq i$ since $f(\sigma, \tau) \in I_{\sigma}$ (by choice of $Y$ ).

We are now ready to prove the missing theorem.

Theorem 2.26. Fix some $n \geq 2$, and suppose that $\mathrm{TS}_{d_{s}+1}^{s}$ strongly preserves 2hyperimmunity for every $0<s<n$, and that $\mathrm{TS}_{d_{n-1}+1}^{n}$ preserves 2-hyperimmunity. Then $\mathrm{TS}_{d_{n}+1}^{n}$ strongly preserves 2-hyperimmunity.

Proof. Fix a coloring $f:[\omega]^{n} \rightarrow d_{n}+1$, and a bifamily $\mathcal{H}$ which is 2-hyperimmune. Let $q=\sum_{0<s<n} d_{s} d_{n-s}$. By Lemma 2.23, we assume that there exists a finite set $I_{f}$ of cardinality $q$ such that for every $0<s<n$,

$$
\left(\forall \sigma \in[\omega]^{s}\right)(\exists b)\left(\forall \tau \in[\omega \cap(b,+\infty)]^{n-s}\right) f(\sigma, \tau) \in I_{f} .
$$

Let $p=1+2 d_{n-1}+\sum_{0 \leq s<n} d_{s-1} d_{n-s-1}$, so $d_{n}=p+q-1$. By renaming the colors of $f$, we can assume without loss of generality that $I_{f}=\left\{p, p+1, \ldots, d_{n}\right\}$. We will construct simultaneously $p$ infinite sets $G_{0}, \ldots, G_{p-1}$ such that $\mathcal{H}$ is $G_{i}$-2hyperimmune for some $i<p$. We furthermore ensure that for each $i<p, G_{i}$ is $f$-thin for color $i$. We construct our sets $G_{0}, \ldots, G_{p-1}$ by a Mathias forcing whose conditions are tuples $\left(F_{0}, \ldots, F_{p-1}, X\right)$, where $\left(F_{i}, X\right)$ is a Mathias condition for each $i<p$ and the following properties hold:

(a) $\left(\forall \sigma \in\left[F_{i}\right]^{s}\right)\left(\forall \tau \in\left[F_{i} \cup X\right]^{n-s}\right) f(\sigma, \tau) \geq p$ for every $0<s<n$.

(b) $F_{i}$ is $f$-thin for color $i$ for every $i<p$.

(c) $\mathcal{H}$ is $X$-2-hyperimmune.

A precondition is a tuple of Mathias conditions satisfying (b)(c). A precondition $d=\left(E_{0}, \ldots, E_{p-1}, Y\right)$ extends a precondition $c=\left(F_{0}, \ldots, F_{p-1}, X\right)($ written $d \leq c)$ if $\left(E_{i}, Y\right)$ Mathias extends $\left(F_{i}, X\right)$ for each $i<p$. Obviously, $(\emptyset, \ldots, \emptyset, \omega)$ is a condition. Therefore, the partial order is non-empty. We note the following simple properties of conditions.

\section{Lemma 2.27 .}

(1) Every precondition can be extended to a condition.

(2) For every condition $c=\left(F_{0}, \ldots, F_{p-1}, X\right)$, every $i<p$ and every finite set $E \subseteq X$ f-thin for color $i, d=\left(F_{0}, \ldots, F_{i-1}, F_{i} \cup E, F_{i+1}, \ldots, F_{p-1}, X\right)$ is a precondition extending $c$.

Proof. Item (1) is trivial by (2.4). For item (2), by property (a)(b) of condition $c$ and $f$-thin for color $i$ of $E, i \notin f\left[F_{i} \cup E\right]^{n}$, so $d$ is a precondition.

The next lemma states that every sufficiently generic filter yields infinite sets $G_{0}, \ldots, G_{p-1}$.

Lemma 2.28. For every condition $c=\left(F_{0}, \ldots, F_{p-1}, X\right)$ and every $i<p$, there is an extension $d=\left(E_{0}, \ldots, E_{p-1}, X\right)$ of $c$ such that $\left|E_{i}\right|>\left|F_{i}\right|$.

Proof. Fix $c$ and some $i<p$, and let $x \in X \backslash F_{i}$. In particular, $[x]^{n}=\emptyset$, so $i \notin f[x]^{n}$. Thus, by Lemma 2.27 , there is an extension $d=\left(E_{0}, \ldots, E_{p-1}, X\right)$ of $c$ such that $E_{i}=F_{i} \cup\{x\}$.

A $p$-tuple of sets $G_{0}, \ldots, G_{p-1}$ satisfies a condition $c=\left(F_{0}, \ldots, F_{p-1}, X\right)$ if $G_{i}$ satisfies the Mathias condition $\left(F_{i}, X\right)$. A condition $c$ forces a formula $\varphi\left(G_{0}, \ldots, G_{p-1}\right)$ 
if the formula holds for every $p$-tuple of sets $G_{0}, \ldots, G_{p-1}$ satisfying $c$. For every $e_{0}, \ldots, e_{p-1} \in \omega$, we want to satisfy the following requirement

$$
\mathcal{R}_{e_{0}, \ldots, e_{p-1}}: \mathcal{R}_{e_{0}} \vee \cdots \vee \mathcal{R}_{e_{p-1}}
$$

where $\mathcal{R}_{e_{i}}$ is the requirement

$$
\text { If } \Phi_{e_{i}}^{G_{i}} \text { is a total, then } \Phi_{e_{i}}^{G_{i}} \text { meets } \mathcal{H} \text {. }
$$

Lemma 2.29. For every condition $c$ and every $p$-tuple of indices $e_{0}, \ldots, e_{p-1}$, there is an extension $d$ of $c$ forcing $\mathcal{R}_{e_{0}, \ldots, e_{p-1}}$.

Proof. Fix $c=\left(F_{0}, \ldots, F_{p-1}, X\right)$. By Lemma 2.27 for notation convenience, we assume $F_{i}=\emptyset$ and $X=\omega 8$. We define a partial computable biarray as follows.

Defining $U_{n}$. Given $r \in \omega$, search computably for some finite set $U_{r}>r$ (if it exists) such that for every pair of colorings $g, h:[\omega]^{n} \rightarrow d_{n}+1$, there is a $n$-TSsufficient $p$-tuple $\left(\mathcal{E}_{i}: i<p\right)$ of finite collections of finite sets with $\mathcal{E}_{i}$ being $g$-thin, $h$-thin for color $i$ such that for every $i<p$, every $E \in \mathcal{E}_{i}$, we have

$$
\Phi_{e_{i}}^{E}(r ; 1) \downarrow \subseteq U_{r} .
$$

Defining $V_{r, m}$. Given $r, m \in \omega$, search computably for some finite set $V_{r, m}>m$ (if it exists) such that for every coloring $g:[\omega]^{n} \rightarrow d_{n}+1$, there is some $i<p$ and some $E_{i} g$-thin for color $i$ such that

$$
\Phi_{e_{i}}^{E_{i}}(r ; 1) \downarrow \subseteq U_{r} \wedge \Phi_{e_{i}}^{E_{i}}(r, m ; 2) \downarrow \subseteq V_{r, m} .
$$

We now have multiple outcomes, depending on which $U_{r}$ and $V_{r, m}$ is found.

- Case 1: $U_{r}$ is not found for some $r \in \omega$. By compactness, the following $\Pi_{1}^{0}$ class $\mathcal{P}$ of pairs of colorings $g, h:[\omega]^{n} \rightarrow d_{n}+1$ is nonempty: there is no $n$-TS-sufficient $\left(\mathcal{E}_{i}: i<p\right)$ finite collections of finite sets such that $\mathcal{E}_{i}$ is both $g$-thin, $h$-thin for color $i$ and for every $i<p, E \in \mathcal{E}_{i}$, we have $\Phi_{e_{i}}^{E}(r ; 1) \downarrow$.

As WKL preserves 2-hyperimmunity (Corollary 2.7), there is a member $g, h$ of $\mathcal{P}$ such that $\mathcal{H}$ is $g \oplus h$-2-hyperimmune. Unfolding the definition of $n$-TS-sufficient and use compactness, the following $\Pi_{1}^{0, g \oplus h}$ class $\mathcal{Q}$ of sequence $\left(f_{s, j}:[\omega]^{s} \rightarrow d_{n}+1\right)_{0 \leq s<n, j<d_{n-s-1}}$ of colorings is nonempty: for every $i<p$, every finite set $E$ which is both $g$-thin, $h$-thin for color $i$ and is $f_{s, j}$-thin for color $i$ for all $0 \leq s<n, j<d_{n-s-1}$, we have $\Phi_{e_{i}}^{E}(r ; 1) \uparrow$.

As WKL preserves 2-hyperimmunity (Corollary 2.7), there is a member $\left(f_{s, j}: 0 \leq s<n, j<d_{n-s-1}\right)$ of $\mathcal{Q}$ such that $\mathcal{H}$ is $g \oplus h \oplus_{0 \leq s<n, j<d_{n-s-1}} f_{s, j^{-}}$ 2-hyperimmune. Since $\mathrm{TS}_{d_{s-1}+1}^{s}$ preserves 2-hyperimmunity for all $0 \leq s \leq$ $n$, there is an infinite set $Y$ such that $\left|f_{s, j}[Y]^{s}\right| \leq d_{s-1}$ for all $0 \leq s<$ $n, j<d_{n-s-1},\left|g[Y]^{s}\right|,\left|h[Y]^{s}\right| \leq d_{n-1}$ and $\mathcal{H}$ is $Y$-2-hyperimmune. Since $p>2 d_{n-1}+\sum_{0<s<n} d_{s-1} d_{n-s-1}$, there is an $i<p$ such that $Y$ is $f_{s, j}$-thin for color $i$ for all $0 \leq s<n, j<d_{n-s-1}$ and both $g$-thin, $h$-thin for color $i$.

Clearly $d=\left(F_{0}, \ldots, F_{p-1}, Y\right)$ is an extension of $c 9^{9}$. We prove that $d$ forces $\mathcal{R}_{e_{0}, \ldots, e_{p-1}}$. This is because if $G_{i}$ satisfies $\left(F_{i}, Y\right)$, then $G_{i}$ is both

\footnotetext{
${ }^{8}$ More specifically, if we can always extends a condition of form $(\emptyset, \cdots, \emptyset, X)$, then given a condition $\left(F_{0}, \ldots, F_{p-1}, X\right)$, we can find a desired extension $\left(E_{0}, \cdots, E_{p-1}\right)$ of $(\emptyset, \cdots, \emptyset, X)$. But $\left(F_{0} \cup E_{0}, \cdots, F_{p-1} \cup E_{p-1}, Y\right)$ is a precondition by Lemma 2.27

${ }^{9}$ When we say "extension of $c$ ", we mean a precondition extending $c$.
} 
$g$-thin and $h$-thin for color $i$ and $f_{s, j}$-thin for color $i$ for all $0 \leq s<n, j<$ $d_{n-s-1}$. Thus, by definition of $g, h,\left(f_{s, j}: 0 \leq s<n, j<d_{n-s-1}\right)$, we have $\Phi_{e_{i}}^{G_{i}}(r ; 1) \uparrow$.

- Case 2: $U_{r}$ is found, but not $V_{r, m}$ for some $r, m \in \omega$. By compactness, the following $\Pi_{1}^{0}$ class $\mathcal{P}$ of colorings $g:[\omega]^{n} \rightarrow d_{n}+1$ is nonempty: for every $i<p$ and every $E_{i} g$-thin for color $i$,

$$
\Phi_{e_{i}}^{E_{i}}(r ; 1) \downarrow \subseteq U_{r} \Rightarrow \Phi_{e_{i}}^{E_{i}}(r, m ; 2) \uparrow .
$$

As WKL preserves 2-hyperimmunity (Corollary 2.7), there is a member $g$ of $\mathcal{P}$ such that $\mathcal{H}$ is $g$-2-hyperimmune. By definition of $U_{r}$ (where we take $h=f)$, there is a $n$-TS-sufficient $p$-tuple $\left(\mathcal{E}_{i}: i<p\right)$ of finite collections of finite sets such that $\mathcal{E}_{i}$ is both $g$-thin and $f$-thin for color $i$ and for every $i<p$, every $E \in \mathcal{E}_{i}$, we have

$$
\Phi_{e_{i}}^{E}(r ; 1) \downarrow \subseteq U_{r} .
$$

By Lemma 2.25, there is an $i<p, E \in \mathcal{E}_{i}$ and an infinite set $Y$ such that $E \cup Y$ is $g$-thin for color $i$ and $\mathcal{H}$ is $Y$-2-hyperimmune. Consider the precondition $\left(F_{0}, \cdots, F_{i-1}, F_{i} \cup E, F_{i+1}, \cdots F_{p-1}, Y\right)$.

It remains to show that $d$ forces $\Phi_{e_{i}}^{G_{i}}(n, m ; 2) \uparrow$. This is because if $G_{i}$ satisfies $(E, Y)$, then $G_{i}$ is $g$-thin for color $i$. But $\Phi_{e_{i}}^{E}(r ; 1) \downarrow \subseteq U_{r}$. Thus, by definition of $g$ (namely 2.5$), \Phi_{e_{i}}^{G_{i}}(r, m ; 2) \uparrow$.

- Case 3: $U_{r}$ and $V_{r, m}$ are found for every $r, m \in \omega$. By 2-hyperimmunity of $\mathcal{H}$, there is some $r, m \in \omega$ such that $\left(U_{r}, V_{r, m}\right) \in \mathcal{H}$. In particular, by definition of $V_{n, m}$ (where we take $g=f$ ), there is some $i<p$ and some $E_{i}$ $f$-thin for color $i$ such that

$$
\Phi_{e_{i}}^{E_{i}}(r ; 1) \downarrow \subseteq U_{r} \wedge \Phi_{e_{i}}^{E_{i}}(r, m ; 2) \downarrow \subseteq V_{r, m}
$$

Since $i \notin f\left[E_{i}\right]^{n}$, we have $d=\left(F_{0}, \ldots, F_{i-1}, F_{i} \cup E_{i}, F_{i+1}, \ldots, F_{p-1}, X\right)$ is an extension of $c$. Clearly $d$ forces $\mathcal{R}_{e_{0}, \ldots, e_{p-1}}$.

This completes the proof of Lemma 2.29 .

Let $\mathcal{F}=\left\{c_{0}, c_{1}, \ldots\right\}$ be a sufficiently generic filter for this notion of forcing, where $c_{s}=\left(F_{0, s}, \ldots, F_{p-1, s}, X_{s}\right)$, and let $G_{i}=\bigcup_{s} F_{i, s}$ for every $i<p$. By property (b) of a condition, for every $i<p, G_{i}$ is $f$-thin for color $i$. By Lemma 2.28 , $G_{0}, \ldots, G_{p-1}$ are all infinite, and by Lemma 2.29 there is some $i<p$ such that $\mathcal{H}$ is $G_{i}$-2-hyperimmune. This completes the proof of Theorem 2.26 .

2.6. $\mathrm{FS}^{2}$ preserves 2-hyperimmunity. The purpose of this section is to prove the following theorem.

Theorem 2.30. FS $^{2}$ preserves 2-hyperimmunity.

We start with a lemma very similar to Lemma 2.14, which establishes a bridge between strong preservation for a principle over $n$-tuples and preservation for the same principle over $(n+1)$-tuples.

Lemma 2.31. Fix some $n \geq 1$. If $\mathrm{FS}^{n}$ strongly preserves 2-hyperimmunity, then $\mathrm{FS}^{n+1}$ preserves 2-hyperimmunity. 
Proof. Let $\mathcal{H}$ be a 2-hyperimmune family, and $f:[\omega]^{n+1} \rightarrow \omega$ be a computable instance of $\mathrm{FS}^{n+1}$. Let $\vec{R}=\left\langle R_{\sigma, i}: \sigma \in[\omega]^{n}, i \in \omega\right\rangle$ be the computable family of sets defined for every $\sigma \in[\omega]^{n}$ and $i \in \omega$ by

$$
R_{\sigma, i}=\{x \in \omega: f(\sigma, x)=i\} .
$$

Since $\mathrm{COH}$ preserves 2-hyperimmunity (Corollary 2.9 ), there is an $\vec{R}$-cohesive set $G$ ${ }^{10}$ such that $\mathcal{H}$ is $G$-2-hyperimmune. Let $g:[\omega]^{n} \rightarrow \omega$ be the instance of $\mathrm{FS}^{n}$ defined for every $\sigma \in[\omega]^{n}$ by

$$
g(\sigma)= \begin{cases}\lim _{x \in G} f(\sigma, x) & \text { if it exists } \\ 0 & \text { otherwise }\end{cases}
$$

By strong preservation of $\mathrm{FS}^{n}$, there is an infinite $g$-free set $H \subseteq G$ such that $\mathcal{H}$ is $G \oplus H$-2-hyperimmune. By thinning out the set $H$, we obtain an infinite $G \oplus H$-computable $f$-free set $\tilde{H} \subseteq H$. In particular, $\mathcal{H}$ is $\tilde{H}$-2-hyperimmune.

We shall define a particular kind of function called left trapped function. The notion of trapped function was introduced by Wang in 21 to prove that FS does not imply ACA over $\omega$-models. It was later reused by the second author in 13,18 .

Definition 2.32. A function $f:[\omega]^{n} \rightarrow \omega$ is left (resp. right) trapped if for every $\sigma \in[\omega]^{n}, f(\sigma) \leq \max \sigma$ (resp. $\left.f(\sigma)>\max \sigma\right)$.

The following lemma is a particular case of a more general statement proven by the second author in 13 . It follows from the facts that $\mathrm{FS}^{n}$ for right trapped functions is strongly computably reducible to the diagonally non-computable principle (DNR), which itself is strongly computably reducible to $\mathrm{FS}^{n}$ for left trapped functions.

Lemma 2.33 (Patey in 13]). For each $n \geq 1$, if $\mathrm{FS}^{n}$ for left trapped functions (strongly) preserves 2-hyperimmune, then so does $\mathrm{FS}^{n}$.

It therefore suffices to prove strong preservation of 2-hyperimmunity for $\mathrm{FS}^{1}$ restricted to left trapped functions. We first prove a technical lemma thinning out colors while preserving 2-hyperimmunity. Fix a set $C$, an infinite set $X \leq_{T} C$, a $C$-2-hyperimmune bifamily $\mathcal{H}$ and a left trapped coloring $f:[\omega]^{n} \rightarrow \omega$.

Lemma 2.34. Assume $\mathrm{FS}^{s}$ strongly preserves 2-hyperimmunity for each $0 \leq s<n$. There exists an infinite set $Y \subseteq X$ such that $\mathcal{H}$ is $Y \oplus C$-2-hyperimmune, and for every $\sigma \in[Y]^{<\omega}$ such that $0 \leq|\sigma|<n$,

$$
(\forall x \in Y \backslash \sigma)(\exists b)\left(\forall \tau \in[Y \cap(b,+\infty)]^{n-|\sigma|}\right) f(\sigma, \tau) \neq x .
$$

Proof. By Proposition 2.19 and since $\mathrm{TS}_{d_{s}+1}^{s}$ strongly preserves 2-hyperimmunity for all $s \in \omega$ (Theorem 2.22), there exists a set $X_{0} \subseteq X$ with $\mathcal{H}$ being $X_{0}$-2hyperimmune such that for all $\sigma \in[\omega]^{<\omega}$ with $|\sigma|<n$, there exists $I_{\sigma}$ with $\left|I_{\sigma}\right| \leq$ $d_{n-|\sigma|}$ such that for every $x \notin I_{\sigma}$,

$$
(\exists b)\left(\forall \tau \in\left[X_{0} \cap(b, \infty)\right]^{n-|\sigma|}\right) f(\sigma, \tau) \neq x .
$$

For each $s<n$ and $i<d_{n-s}$, let $f_{s, i}:[\omega]^{s} \rightarrow \omega$ be the coloring such that $f_{s, i}(\sigma)$ is the $i$ th element of $I_{\sigma}$. By strong preservation of $\mathrm{FS}^{s}$ for each $0 \leq s<n$, there is

${ }^{10}$ Here $G$ is $\vec{R}$-cohesive iff for every $\sigma \in[\omega]^{n}$ : either $\lim _{x \in G} f(\sigma, x)$ exists, or $\{f(\sigma, x)=i: x \in G\}$ is finite for all $i \in \omega$. We are not using exactly Corollary 2.9 but a similar proof applies here. 
an infinite set $Y \subseteq X_{0}$ such that $Y$ is $f_{s, i}$-free for all $0 \leq s<n, i<d_{n-s}$ and $\mathcal{H}$ is $Y$-2-hyperimmune.

We prove that $Y$ is desired. Fix $s<n, \sigma \in[Y]^{s}$ and $x \in Y \backslash \sigma$. If $(\forall b)(\exists \tau \in$ $\left.[Y \cap(b,+\infty)]^{n-s}\right) f(\sigma, \tau)=x$, then by choice of $X_{0}$ (and $Y \subseteq X_{0}$ ), there exists an $i<d_{n-s}$ such that $f_{s, i}(\sigma)=x$, contradicting $f_{s, i}$-freeness of $Y$. So $(\exists b)(\forall \tau \in$ $\left.[Y \cap(b,+\infty)]^{n-s}\right) f(\sigma, \tau) \neq x$.

Theorem 2.35. FS $^{1}$ for left trapped functions strongly preserves 2-hyperimmunity.

Proof. Fix some 2-hyperimmune bifamily $\mathcal{H}$, and a left trapped coloring $f: \omega \rightarrow \omega$. By Lemma 2.34, we assume

$$
(\forall x \in \omega)(\exists b)(\forall y \in(b,+\infty)) f(y) \neq x .
$$

We will construct an infinite $f$-free set $G$ such that $\mathcal{H}$ is $G$-2-hyperimmune by forcing. Our forcing conditions are Mathias conditions $(F, X)$ such that

(a) $\mathcal{H}$ is $X$-2-hyperimmune

(b) $(\forall x \in F \cup X) f(x) \notin F \backslash\{x\}$.

A Mathias condition $(F, X)$ is a precondition if it satisfies (a) and $F$ is $f$-free. Clearly $(\emptyset, \omega)$ is a condition. It's easy to see that:

\section{Lemma 2.36.}

(1) Every precondition can be extended to a condition.

(2) For every condition $(F, X)$, every finite $f$-free set $E \subseteq X$ with $E>F$, $(F \cup E, X)$ is a precondition.

Proof. Item (1) follows from (2.6). For item (2): Since $f$ is left trapped, for every $x \in F, f(x) \notin E$. Combined with $f$-freeness of $F, f(x) \notin(F \cup E) \backslash\{x\}$. Since $E$ is $f$-free, for every $x \in E, f(x) \notin E \backslash\{x\}$. Combined with property (b) of a condition, $f(x) \notin(F \cup E) \backslash\{x\}$.

Lemma 2.37. For every condition $(F, X)$ there exists an extension $(E, Y)$ such that $|E|>|F|$.

Proof. Pick any $x \in X$ with $x>F$. Clearly $\{x\}$ is $f$-free. Thus the conclusion follow from Lemma 2.36 .

For every $e \in \omega$, we want to satisfy the requirement

$$
\mathcal{R}_{e} \text { : If } \Phi_{e}^{G} \text { is total, then } \Phi_{e}^{G} \text { meets } \mathcal{H} \text {. }
$$

Lemma 2.38. For every condition $c$ and every index $e$, there is an extension $d$ of c forcing $\mathcal{R}_{e}$.

Proof. Fix a condition $c=(F, X)$. By Lemma 2.36, for notation convenience, assume $F=\emptyset, X=\omega$. We define a partial computable biarray as follows.

Defining $U_{n}$. Given $n \in \omega$, search computably for some finite set $U_{n}>n$ (if it exists) such that for every pair of left trapped colorings $g, h: \omega \rightarrow \omega$, there is a pair of disjoint finite sets $E_{0}, E_{1}$ which are both $g$-free and $h$-free such that for each $i<2$,

$$
\Phi_{e}^{E_{i}}(n ; 1) \downarrow \subseteq U_{n}
$$


Defining $V_{n, m}$. Given $n, m \in \omega$, search computably for some finite set $V_{n, m}>m$ (if it exists) such that for every left trapped coloring $g: \omega \rightarrow \omega$, there is some $g$-free finite set $E$ such that

$$
\Phi_{e}^{E}(n ; 1) \downarrow \subseteq U_{n} \wedge \Phi_{e}^{E}(n, m ; 2) \downarrow \subseteq V_{n, m}
$$

We now have multiple outcomes, depending on which $U_{n}$ and $V_{n, m}$ is found.

- Case 1: $U_{n}$ is not found for some $n \in \omega$. By compactness, the following $\Pi_{1}^{0}$ class $\mathcal{P}$ of pairs of left trapped colorings $g, h: \omega \rightarrow \omega$ is nonempty: for every pair of pairwise disjoint finite sets $E_{0}, E_{1}$ which are both $g$-free and $h$-free, there is some $i<2$ such that $\Phi_{e}^{E_{i}}(n ; 1) \uparrow$.

As WKL preserves 2-hyperimmunity (Corollary 2.7), there is a member $g, h$ of $\mathcal{P}$ such that $\mathcal{H}$ is $g \oplus h$-2-hyperimmune. There is an infinite $g \oplus h$ computable set $Y$ which is both $g$-free and $h$-free. Let $E_{0}$ (if exists) be $g$-free, $h$-free and $\Phi_{e}^{E_{0}}(n ; 1) \downarrow$; let $b=\max E_{0}$ (or $b=0$ if $E_{0}$ does not exist). Clearly condition $d=(F, Y \backslash[0, b])$ is an extension of $c$. For every $G$ satisfying $d, G$ is both $g$-free and $h$-free, so $\Phi_{e}^{G}(n ; 1) \uparrow$. Thus $d$ forces $\mathcal{R}_{e}$.

- Case 2: $U_{n}$ is found, but not $V_{n, m}$ for some $n, m \in \omega$. By compactness, the following $\Pi_{1}^{0}$ class $\mathcal{P}$ of left trapped colorings $g: \omega \rightarrow \omega$ is nonempty: for every $g$-free set $E$,

$$
\Phi_{e}^{E}(n ; 1) \downarrow \subseteq U_{n} \Rightarrow \Phi_{e}^{E}(n, m ; 2) \uparrow .
$$

As WKL preserves 2-hyperimmunity (Corollary 2.7), there is a member $g$ of $\mathcal{P}$ such that $\mathcal{H}$ is $g$-2-hyperimmune. There is an infinite $g$-computable $g$-free set $Y$. By definition of $U_{n}$ (where we take $h=f$ ), there is a pair of disjoint finite sets $E_{0}, E_{1}$ which are both $g$-free and $f$-free, and such that for every $i<2$,

$$
\Phi_{e}^{E_{i}}(n ; 1) \downarrow \subseteq U_{n} .
$$

Consider the 2-partition $A_{0} \sqcup A_{1}$ of $\omega$ defined by $x \in A_{i}$ if $g(x) \in E_{i}$ and $x \in A_{0}$ if $g(x) \notin \cup_{i<2} E_{i}$. Since $\mathrm{TS}_{2}^{1}$ is computably true, hence preserves 2hyperimmunity, there is an $i<2$ and an infinite set $E_{i}<\tilde{Y} \subseteq Y$ such that $\mathcal{H}$ is $\tilde{Y}$-2-hyperimmune and $g(\tilde{Y}) \cap E_{i}=\emptyset$. We claim that $E_{i} \cup \tilde{Y}$ is $g$-free. Indeed, $E_{i}$ and $\tilde{Y}$ are both $g$-free; since $g$ is left trapped, $g\left(E_{i}\right) \cap \tilde{Y}=\emptyset$; and by choice of $\tilde{Y}, g(\tilde{Y}) \cap E_{i}=\emptyset$.

By $f$-free of $E_{i}, d=\left(E_{i}, \tilde{Y}\right)$ is a precondition extending $c$. We prove that the $d$ forces $\Phi_{e}^{G}(n, m ; 2) \uparrow$. Because $\Phi_{e}^{E_{i}}(n ; 1) \downarrow \subseteq U_{n}$ and $E_{i} \cup \tilde{Y}$ is $g$-free (so every $G$ satisfying $\left(E_{i}, \tilde{Y}\right)$ is $g$-free), by definition of $g$ (namely (2.7) $), d$ forces $\Phi_{e}^{G}(n, m ; 2) \uparrow$.

- Case 3: $U_{n}$ and $V_{n, m}$ are found for every $n, m \in \omega$. By 2-hyperimmunity of $\mathcal{H}$, there is some $n, m \in \omega$ such that $\left(U_{n}, V_{n, m}\right) \in \mathcal{H}$. In particular, by definition of $V_{n, m}$ (where we take $g=f$ ), there is some $f$-free finite set $E$ such that

$$
\Phi_{e}^{E}(n ; 1) \downarrow \subseteq U_{n} \wedge \Phi_{e}^{E}(n, m ; 2) \downarrow \subseteq V_{n, m} .
$$

Clearly $(E, X)$ is a precondition extending $c$ and forcing $\mathcal{R}_{e}$.

This completes the proof of Lemma 2.38 . 
Let $\mathcal{F}=\left\{c_{0}, c_{1}, \ldots\right\}$ be a sufficiently generic filter for this notion of forcing, where $c_{s}=\left(F_{s}, X_{s}\right)$, and let $G=\bigcup_{s} F_{s}$. By property (b) of a condition, $G$ is $f$-free. By Lemma 2.37, $G$ is infinite, and by Lemma 2.38, $\mathcal{H}$ is $G$-2-hyperimmune. This completes the proof of Theorem 2.35 .

2.7. $\mathrm{FS}^{n}$ preserves 2-hyperimmunity. The purpose of this section is to prove the following theorem.

Theorem 2.39. For every $n \geq 1, \mathrm{FS}^{n}$ strongly preserves 2-hyperimmunity.

Proof. We prove by induction over $n \geq 1$ that $\mathrm{FS}^{n}$ preserves and strongly preserves 2-hyperimmunity. If $n=1, \mathrm{FS}^{1}$ is a computably true statement, that is, every instance has a solution computable in the instance, so $\mathrm{FS}^{1}$ preserves 2-hyperimmunity. If $n>1$, then by induction hypothesis, $\mathrm{FS}^{n-1}$ strongly preserves 2-hyperimmunity, so by Lemma 2.31, $\mathrm{FS}^{n}$ preserves 2-hyperimmunity. Assuming by induction hypothesis that $\mathrm{FS}^{t}$ strongly preserves 2-hyperimmunity for every $t<n$, and that $\mathrm{FS}^{n}$ preserves 2-hyperimmunity, then by Theorem 2.43. FS ${ }^{n}$ for left trapped functions strongly preserves 2-hyperimmunity. By Lemma 2.33 , if $\mathrm{FS}^{n}$ for left trapped functions strongly preserves 2-hyperimmunity, so does $\mathrm{FS}^{n}$. This completes the proof.

We first need to prove a technical lemma which will ensure that the reservoirs of our forcing conditions will have good properties, so that the conditions will be extensible. Fix a set $C$, a $C$-2-hyperimmune bifamily $\mathcal{H}$; a finite set $F$ and an infinite set $X \leq_{T} C$; fix a left trapped coloring $f:[\omega]^{n} \rightarrow \omega$.

Lemma 2.40. Suppose that $\mathrm{FS}^{s}$ strongly preserves 2-hyperimmunity for each $0<$ $s<n$. Then there exists an infinite set $Y \subseteq X$ such that $\mathcal{H}$ is $Y \oplus C$-2-hyperimmune, and for each $0<s<n$,

$$
\left(\forall \sigma \in[F]^{s}\right)\left(\forall \tau \in[Y]^{n-s}\right) f(\sigma, \tau) \notin Y \backslash \tau .
$$

Proof. For each $0<s<n$, each $\sigma \in[F]^{s}$ consider the coloring $f_{\sigma}:[\omega]^{n-s} \rightarrow \omega$ defined as $f_{\sigma}(\tau)=f(\sigma, \tau)$. Since $\mathrm{FS}^{s}$ strongly preserves 2-hyperimmunity for each $0<s<n$, there is an infinite set $Y \subseteq X$ such that $\mathcal{H}$ is $Y$-2-hyperimmune and $Y$ is $f_{\sigma}$-free for all $0<s<n, \sigma \in[F]^{s}$. Unfolding the definition of free, $Y$ is desired.

We need to prove a second lemma saying that if we have sufficiently many finite free sets, one of them is extendible into an infinite one. This generalizes the argument of case 2 of Lemma 2.38, where we showed that for every coloring $g: \omega \rightarrow \omega$ and every pair of disjoint $g$-free finite sets $E_{0}, E_{1}$, there is an $i<2$ and an infinite set $Y$ such that $E_{i} \cup Y$ is $g$-free.

Definition 2.41 (FS-sufficient). A collection $\mathcal{F}$ of finite sets is $n$-FS-sufficient iff for every sequence $\left(f_{s, i}:[\omega]^{s} \rightarrow \omega\right)_{s<n, i<d_{n-s-1}}$ of left trapped colorings, there exists an $F \in \mathcal{F}$ such that $F$ is $f_{s, i}$-free for all $s<n, i<d_{n-s-1}$.

In particular, for $n=1$, a collection $\mathcal{F}$ of finite sets is 1-FS-sufficient iff for every left-trapped coloring $f_{0,0}:[\omega]^{0} \rightarrow \omega$, there exists an $F \in \mathcal{F}$ such that $F$ is $f_{0,0}$-free. Note that a coloring $[\omega]]^{\omega} \rightarrow \omega$ is nothing but the choice of an element in $\omega$, which means that for every $x \in \omega$, there exists an $F \in \mathcal{F}$ such that $x \notin F$. This is true as long as $\mathcal{F}$ contains two disjoint sets.

Let $\mathcal{F}$ be a collection of $f$-free finite sets. 
THE REVERSE MATHEMATICS OF THE THIN SET AND THE ERDŐS-MOSER THEOREM 3

Lemma 2.42. Assume that $\mathrm{FS}^{n}$ preserves 2-hyperimmunity and that $\mathrm{FS}^{s}$ strongly preserves 2-hyperimmunity for each $0<s<n$. Suppose $f \leq_{T} C, \mathcal{F}$ is $n$-FSsufficient and $f$-fre 11 . Then there is an $F \in \mathcal{F}$ and an infinite subset $Y \subseteq X$ such that $F \cup Y$ is $f$-free and $\mathcal{H}$ is $C \oplus Y$-2-hyperimmune.

Proof. Using a version of Proposition 2.19 as in Lemma 2.34 but for preservation instead of strong preservation since $f \leq_{T} C$, there is an infinite set $X_{0} \subseteq X$ with $\mathcal{H}$ being $X_{0}$-2-hyperimmune such that for every $\sigma \in[\omega]<\omega$ with $|\sigma|<n$, there is a $I_{\sigma}$ with $\left|I_{\sigma}\right| \leq d_{n-|\sigma|-1}$ such that for every $x \notin I_{\sigma}$,

$$
(\exists b)\left(\forall \tau \in\left[X_{0} \cap(b, \infty)\right]^{n-|\sigma|}\right) f(\sigma, \tau) \neq x .
$$

For each $s<n, i<d_{n-s-1}$, consider the coloring $f_{s, i}:[\omega]^{s} \rightarrow \omega$ such that $f_{s, i}(\sigma)$ is the $i$ th element of $I_{\sigma}$.

Since $\mathcal{F}$ is $n$-FS-sufficient, there is an $F \in \mathcal{F}$ such that $F$ is $f_{s, i}$-free for all $s<n, i<d_{n-s-1}$. By choice of $X_{0}$, let $b \in \omega$ so that for every $\sigma \in[F]^{<\omega}$ with $|\sigma|<n$,

$$
\left(\forall \tau \in\left[X_{0} \cap(b, \infty)\right]^{n-|\sigma|}\right) f(\sigma, \tau) \notin F \backslash I_{\sigma} .
$$

By preservation of 2-hyperimmune of $\mathrm{FS}^{n}$, let infinite $f$-free set $X_{1} \subseteq X_{0} \cap(b, \infty)$ so that $\mathcal{H}$ is $X_{1}$-2-hyperimmune. By Lemma 2.40, let infinite set $Y \subseteq X_{1}$ so that $\mathcal{H}$ is $Y$-2-hyperimmune, $Y>F$ and for every $0<s<n$,

$$
\left(\forall \sigma \in[F]^{s}\right)\left(\forall \tau \in[Y]^{n-s}\right) f(\sigma, \tau) \notin Y \backslash \tau .
$$

We show that $F \cup Y$ is $f$-free. To see this, let $\sigma \in[F]^{<\omega}, \tau \in[Y]^{<\omega}$ with $|\sigma \cup \tau|=n$, we need to prove $f(\sigma, \tau) \notin F \backslash \sigma$ and $f(\sigma, \tau) \notin Y \backslash \tau$. To see $f(\sigma, \tau) \notin$ $Y \backslash \tau$, when $|\tau|=n$, the conclusion follows by $f$-free of $Y$; when $0<|\tau|<n$, the conclusion follows from (2.9); when $|\tau|=0$, the conclusion follows by left trap of $f$. To see $f(\sigma, \tau) \notin F \backslash \sigma$, when $|\sigma|=n$, the conclusion follows from $f$-freeness of $F$. When $|\sigma|<n$, suppose $f(\sigma, \tau) \in F$ (otherwise we are done). By (2.8), $f(\sigma, \tau)=x \in F \cap I_{\sigma}$. Since $x \in I_{\sigma}$, it means for $s=n-|\sigma|$, for some $i<d_{n-s-1}$, $f_{s, i}(\sigma)=x$. In particular, $f_{s, i}(\sigma) \in F$. But $F$ is $f_{s, i}$-free, so $x \notin \sigma$. Thus we are done.

We are now ready to prove the missing theorem.

Theorem 2.43. For each $n \geq 1$, if $\mathrm{FS}^{s}$ strongly preserves 2-hyperimmunity for each $0 \leq s<n$ and $\mathrm{FS}^{n}$ preserves 2-hyperimmunity, then $\mathrm{FS}^{n}$ for left trapped functions strongly preserves 2-hyperimmunity.

Proof. Fix a left trapped coloring $f:[\omega]^{n} \rightarrow \omega$. By Lemma 2.34, we assume that for every $s<n$, every $\sigma \in[\omega]^{s}$,

$$
(\forall x \in \omega \backslash \sigma)(\exists b)\left(\forall \tau \in[\omega \cap(b,+\infty)]^{n-s}\right) f(\sigma, \tau) \neq x .
$$

We will construct an infinite $f$-free set $G$ such that $\mathcal{H}$ is $G$-2-hyperimmune. Our forcing conditions are Mathias conditions $(F, X)$ such that

(a) $\mathcal{H}$ is $X$-2-hyperimmune.

(b) $\left(\forall \sigma \in[F \cup X]^{n}\right) f(\sigma) \notin F \backslash \sigma$.

(c) $\left(\forall \sigma \in[F]^{s}\right)\left(\forall \tau \in[X]^{n-s}\right) f(\sigma, \tau) \notin X \backslash \tau$ for each $0<s<n$.

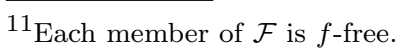


Clearly $(\emptyset, \omega)$ is a condition. A precondition $(F, X)$ is a Mathias condition satisfying (a) and $F$ is $f$-free.

\section{Lemma 2.44.}

(1) Every precondition can be extended to a condition.

(2) For every condition $(F, X)$, every $f$-free set $Y \subseteq X$ with $Y>F, F \cup Y$ is $f$-free.

(3) For every condition $(F, X)$, every finite $f$-free set $E \subseteq X$ with $E>F$, $(F \cup E, X)$ is a precondition.

Proof. For item (1): Fix a precondition $(F, X)$. By 2.10 and $f$-freeness of $F$, there is a $b \in \omega$ so that for every $\sigma \in[F]^{\leq n}$,

$$
\left(\forall \tau \in[\omega \cap(b,+\infty)]^{n-|\sigma|}\right) f(\sigma, \tau) \notin F \backslash \sigma,
$$

which verifies that $(F, X \cap(b, \infty))$ satisfies property (b). By Lemma 2.40, there is a reservoir-extension ${ }^{12}$ of $(F, X \cap(b, \infty))$ satisfying property (c) while preserving property (a). Thus we are done (property (b) is preserved by reservoir-extension).

For item (2): Let $\sigma \in[F]^{<\omega}, \tau \in[Y]^{<\omega}$ with $|\sigma \cup \tau|=n$. We need to show that $f(\sigma, \tau) \notin F \backslash \sigma$ and $f(\sigma, \tau) \notin Y \backslash \tau$. It follows from property (b) of $(F, X)$ that $f(\sigma, \tau) \notin F \backslash \sigma$. To see $f(\sigma, \tau) \notin Y \backslash \tau$, the conclusion follows from property (c) of $(F, X)$ when $|\sigma|,|\tau|>0$; the conclusion follows by left trap of $f$ when $|\tau|=0$; the conclusion follows from $f$-freeness of $Y$ when $|\tau|=n$.

Item (3) follows from item (2) directly.

Lemma 2.45. For every condition $(F, X)$ there exists an extension $(E, Y)$ such that $|E|>|F|$.

Proof. Pick any $x \in X$ so that $x>E$ and set $E=F \cup\{x\}$. Since $\{x\}$ is $f$-free, so by Lemma 2.44. $(E, X)$ is a precondition.

For every $e \in \omega$, we want to satisfy the requirement

$$
\mathcal{R}_{e} \text { : If } \Phi_{e}^{G} \text { is a total, then } \Phi_{e}^{G} \text { meets } \mathcal{H} .
$$

Lemma 2.46. For every condition $c$ and every index $e$, there is an extension $d$ of $c$ forcing $\mathcal{R}_{e}$.

Proof. Fix $c=(F, X)$. By Lemma 2.44 for notation convenience, assume $F=\emptyset$ and $X=\omega$. We define a partial computable biarray as follows.

Defining $U_{n}$. Given $r \in \omega$, search computably for some finite set $U_{r}>r$ (if it exists) such that for every pair of left trapped colorings $g, h:[\omega]^{n} \rightarrow \omega$, there is a finite $n$-FS-sufficient collection $\mathcal{E}$ of finite sets which is both $g$-free and $h$-free such that for every $E \in \mathcal{E}$,

$$
\Phi_{e}^{E}(r ; 1) \downarrow \subseteq U_{r}
$$

Defining $V_{r, m}$. Given $r, m \in \omega$, search computably for some finite set $V_{r, m}>m$ (if it exists) such that for every left trapped coloring $g:[\omega]^{n} \rightarrow \omega$, there is some $g$-free finite set $E$ such that

$$
\Phi_{e}^{E}(r ; 1) \downarrow \subseteq U_{r} \wedge \Phi_{e}^{E}(r, m ; 2) \downarrow \subseteq V_{r, m} .
$$

We now have multiple outcomes, depending on which $U_{r}$ and $V_{r, m}$ is found.

\footnotetext{
${ }^{12} \mathrm{~A}$ Mathias condition $(E, Y)$ reservoir-extends $(F, X)$ if it extends $(F, X)$ and $E=F$.
} 
THE REVERSE MATHEMATICS OF THE THIN SET AND THE ERDŐS-MOSER THEOREM\&5

- Case 1: $U_{r}$ is not found for some $r \in \omega$. By compactness, the following $\Pi_{1}^{0}$ class $\mathcal{P}$ of pairs of left trapped colorings $g, h:[\omega]^{n} \rightarrow \omega$ is nonempty: there is no $n$-FS-sufficient finite collection $\mathcal{E}$ of finite sets which are both $g$-free and $h$-free, such that for every $E \in \mathcal{E}$, we have $\Phi_{e}^{E}(r ; 1) \downarrow$.

As WKL preserves 2-hyperimmunity (Corollary 2.7), there is a member $g, h$ of $\mathcal{P}$ such that $\mathcal{H}$ is $g \oplus h$-2-hyperimmune. Unfolding the definition of $n$ FS-sufficient and use compactness, the following $\Pi_{1}^{0, g \oplus h}$ class $\mathcal{Q}$ of sequence $\left(f_{s, i}:[\omega]^{s} \rightarrow \omega\right)_{s<n, i<d_{n-s-1}}$ of left trapped colorings is nonempty:

for every finite set $E$ which is $g$-free, $h$-free and

$$
f_{s, i} \text {-free for each } s<n, i<d_{n-s-1}, \Phi_{e}^{E}(r ; 1) \uparrow .
$$

As WKL preserves 2-hyperimmunity (Corollary 2.7), there is a member $\left(f_{s, i}: s<n, i<d_{n-s-1}\right)$ of $\mathcal{Q}$ such that $\mathcal{H}$ is $g \oplus h \oplus_{s<n, i<d_{n-s-1}} f_{s, i}-2-$ hyperimmune. As $\mathrm{FS}^{n}$ preserves 2-hyperimmunity, there is an infinite set $Y$ which is both $g$-free, $h$-free and $f_{s, i}$-free for each $s<n, i<d_{n-s-1}$ and such that $\mathcal{H}$ is $Y$-2-hyperimmune. Clearly for every $G$ satisfying condition $(F, Y), G$ is $g$-free, $h$-free and $f_{s, i}$-free for each $s<n, i<d_{n-s-1}$, so $\Phi_{e}^{G}(r ; 1) \uparrow$. i.e., The condition $d=(F, Y)$ is an extension of $c$ forcing $\mathcal{R}_{e}$.

- Case 2: $U_{r}$ is found, but not $V_{r, m}$ for some $r, m \in \omega$. By compactness, the following $\Pi_{1}^{0}$ class $\mathcal{P}$ of left trapped colorings $g:[\omega]^{n} \rightarrow \omega$ is nonempty: for every $g$-free set $E$,

$$
\Phi_{e}^{E}(r ; 1) \downarrow \subseteq U_{r} \Rightarrow \Phi_{e}^{E}(r, m ; 2) \uparrow .
$$

As WKL preserves 2-hyperimmunity (Corollary 2.7), there is a member $g$ of $\mathcal{P}$ such that $\mathcal{H}$ is $g$-2-hyperimmune. By definition of $U_{r}$ (where we take letting $h=f$ ), there is a $n$-FS-sufficient finite collection $\mathcal{E}$ of finite sets which is both $g$-free and $f$-free and such that for each $E \in \mathcal{E}$,

$$
\Phi_{e}^{E}(r ; 1) \downarrow \subseteq U_{r} .
$$

By Lemma 2.42, there is an infinite set $Y$ and some $E \in \mathcal{E}$ such that $\mathcal{H}$ is $Y$-2-hyperimmune and $E \cup Y$ is $g$-free. Consider the precondition $d=(E, Y)$. It remains to prove that $d$ forces $\Phi_{e}^{G}(r, m ; 2) \uparrow$. Since $E \cup Y$ is $g$-free, so every $G$ satisfying $(E, Y)$ is $g$-free. By definition of $g$ (namely $2.12)$ and $\Phi_{e}^{E}(r ; 1) \downarrow \subseteq U_{r}$, for every $G$ satisfying $(E, Y), \Phi_{e}^{G}(r, m ; 2) \uparrow$.

- Case 3: $U_{r}$ and $V_{r, m}$ are found for every $r, m \in \omega$. By 2-hyperimmunity of $\mathcal{H}$, there is some $r, m \in \omega$ such that $\left(U_{r}, V_{r, m}\right) \in \mathcal{H}$. In particular, by definition of $V_{r, m}$ (where we take $g=f$ ), there is some $f$-free finite set $E$ such that

$$
\Phi_{e}^{E}(r ; 1) \downarrow \subseteq U_{r} \wedge \Phi_{e}^{E}(r, m ; 2) \downarrow \subseteq V_{r, m} .
$$

Consider the precondition $(E, X)$. Clearly it forces $\mathcal{R}_{e}$.

This completes the proof of Lemma 2.46 .

Let $\mathcal{F}=\left\{c_{0}, c_{1}, \ldots\right\}$ be a sufficiently generic filter for this notion of forcing, where $c_{s}=\left(F_{s}, X_{s}\right)$, and let $G=\bigcup_{s} F_{s}$. By property (b) of a condition, $G$ is $f$ free. By Lemma 2.45, $G$ is infinite, and by Lemma 2.46, $\mathcal{H}$ is $C \oplus G$-2-hyperimmune. This completes the proof of Theorem 2.43 . 


\section{ERDŐS-MOSER THEOREM HAS NO UNIVERSAL INSTANCE}

In this section, we prove Theorem 3.6, that EM does not have a universal instance. To this end, we construct a pair of computable EM instances $T_{0}, T_{1}$ such that for every computable EM instance $T, T$ admits a solution that does not compute any solution to either $T_{0}$ or $T_{1}$. Given a EM instance $T$ and two sets $A, B$, we write $A \rightarrow_{T} B$ iff for every $x \in A, y \in B, T(x, y)$; we say $T$ diagonalizes against $(A, B)$ if: $A \rightarrow_{T} B$ and for all but finitely many $x \in \omega, B \rightarrow_{T} x \rightarrow_{T} A$. The point of $T$ diagonalizes against $(A, B)$ is that if a set $H$ has nonempty intersection with both $A, B$, then there is no solution to $T$ containing $H$.

\section{Definition 3.1.}

(1) A 4-array is a sequence of 4-tuple of finite sets (of integers) $\left\langle E_{n}, E_{n, m, l}, F_{n, m}, F_{n, m, l}\right.$ : $n, m, l \in \omega\rangle$ such that for every $n, m, l \in \omega, E_{n}>n, E_{n, m, l}>m, F_{n, m}>n$ and $F_{n, m, l}>l$.

(2) A pair of EM instances $\left(T_{0}, T_{1}\right)$ is $C$-4-hyperimmune if for every $C$-computable 4-array $\left\langle E_{n}, E_{n, m, l}, F_{n, m}, F_{n, m, l}: n, m, l \in \omega\right\rangle$, there exist $n, m, l \in \omega$ such that $T_{0}$ diagonalizes against $\left(E_{n}, E_{n, m, l}\right)$ and $T_{1}$ diagonalizes against $\left(F_{n, m}, F_{n, m, l}\right)$.

For notation convenience, in this section we regard each Turing machine $\Phi$ as computing a 4 -array. We will therefore assume that whenever $\Phi(n ; 1)$ converges, then it will output (the canonical index of) a finite set $E_{n}>n$. Similarly for $\Phi(n, m, l ; 2), \Phi(n, m ; 3)$ and $\Phi(n, m ; 4)$ with the appropriate lower bound.

By finite injury argument (as Proposition 2.10, we have:

Proposition 3.2. There exists a pair of computable stable 4-hyperimmune EM instance.

Proof. We build the tournaments $T_{0}$ and $T_{1}$ by a finite injury priority argument. For simplicity, we see $T_{0}$ and $T_{1}$ as functions over $f_{0}, f_{1}:[\omega]^{2} \rightarrow 2$ by letting for every $x<y$ and $i<2, T_{i}(x, y)$ hold iff $f_{i}(x, y)=0$. For every $e \in \omega$, we want to satisfy the following requirement:

$$
\begin{aligned}
\mathcal{R}_{e}: \text { If } \Phi_{e} \text { is total, then there is some } n, m, l \in \omega \text { such that } \\
\\
T_{0} \text { diagonalizes against }\left(\Phi_{e}(n ; 1), \Phi_{e}(n, m, l ; 2)\right) \text { and } \\
T_{1} \text { diagonalizes against }\left(\Phi_{e}(n, m ; 3), \Phi_{e}(n, m, l ; 4)\right) .
\end{aligned}
$$

The requirements are given the usual priority ordering $\mathcal{R}_{0}<\mathcal{R}_{1}<\ldots$ Initially, the requirements are neither partially, nor fully satisfied.

(i) A requirement $\mathcal{R}_{e}$ requires a first attention at stage $s$ if it is not first satisfied and $\Phi_{e, s}(n ; 1) \downarrow=E_{n}$ for some set $E_{n} \subseteq\{e+1, \ldots, s-1\}$ such that no element in $E_{n}$ is restrained by a requirement of higher priority. If it receives attention, then it puts a restrain on $E_{n}$, commit the elements of $E_{n}$ to be in $C_{0}\left(f_{0}\right)$, and is declared first satisfied.

(ii) A requirement $\mathcal{R}_{e}$ requires a second attention at stage $s$ if it is not second satisfied and $\Phi_{e, s}(n ; 1) \downarrow=E_{n}$ and $\Phi_{e, s}(n, m ; 3) \downarrow=F_{n, m}$ for some sets $E_{n}, F_{n, m} \subseteq\{e+1, \ldots, s-1\}$ such that no element in $E_{n} \cup F_{n, m}$ is restrained by a requirement of higher priority and such that $f_{0}(x, y)=0$ for every $x \in E_{n}$ and $y \in\{m+1, m+2, \ldots, s-1\}$. If it receives attention, then it puts a restrain on $E_{n} \cup F_{n, m}$, commit the elements of $E_{n}$ to be in $C_{0}\left(f_{0}\right)$ 
and the elements of $F_{n, m}$ to be in $C_{0}\left(f_{1}\right)$. Then the requirement is declared second satisfied.

(iii) A requirement $\mathcal{R}_{e}$ requires a third attention at stage $s$ if it is not fully satisfied, and $\Phi_{e, s}(n ; 1) \downarrow=E_{n}, \Phi_{e, s}(n, m, l ; 2) \downarrow=E_{n, m, l}, \Phi_{e, s}(n, m ; 3) \downarrow=$ $F_{n, m}$ and $\Phi_{e, s}(n, m, l ; 4) \downarrow=F_{n, m, l}$ for some sets $E_{n}, E_{n, m, l}, F_{n, m}, F_{n, m, l} \subseteq$ $\{e+1, \ldots, s-1\}$ which are not restrained by a requirement of higher priority, and such that $f_{0}(x, y)=0$ for every $x \in E_{n}$ and $y \in\{m+1, m+2, \ldots, s-1\}$, and $f_{1}(x, y)=0$ for every $x \in F_{n, m}$ and $y \in\{l+1, l+2, \ldots, s-1\}$. If it receives attention, then it puts a restrain on $E_{n} \cup E_{n, m, l} \cup F_{n, m} \cup F_{n, m, l}$, commit the elements of $E_{n}$ to be in $C_{1}\left(f_{0}\right)$, the elements of $E_{n, m, l}$ to be in $C_{0}\left(f_{0}\right)$, the elements of $F_{n, m}$ to be in $C_{1}\left(f_{1}\right)$, the elements of $F_{n, m, l}$ to be in $C_{0}\left(f_{1}\right)$, and is declared fully satisfied.

At stage 0 , we let $f_{0}=f_{1}=\emptyset$. Suppose that at stage $s$, we have defined $f_{0}(x, y)$ and $f_{1}(x, y)$ for every $x<y<s$. For every $x<s$ and $i<2$, if it is committed to be in some $C_{j}\left(f_{i}\right)$, set $f_{i}(x, s)=j$, and otherwise set $f_{i}(x, s)=0$. Let $\mathcal{R}_{e}$ be the requirement of highest priority which requires attention. If $\mathcal{R}_{e}$ requires a third attention, then execute the third procedure. Otherwise, if it requires the second attention, then execute the second procedure, and in the last case, execute the first one. In any case, reset all the requirements of lower priorities by setting them unsatisfied, releasing all their restrains, and go to the next stage. This completes the construction. On easily sees by induction that each requirement acts finitely often, and is eventually fully satisfied. This procedure also yields stable colorings, hence stable tournaments.

Before proving our core argument which will be Theorem 1.7, we prove a few preservation results. These results will be used to assume some good properties on our tournaments.

Proposition 3.3. $\mathrm{COH}$ preserves 4-hyperimmunity. i.e., For every set $C$ and $C$ 4-hyperimmune EM instance pair $\left(T_{0}, T_{1}\right)$, every $C$-computable $\mathrm{COH}$ instance $\vec{R}$, there exists a solution $G$ of $\vec{R}$ such that $\left(T_{0}, T_{1}\right)$ is $C \oplus G$-4-hyperimmune.

Proof. Let $\mathcal{B}$ be the class of all 4 -arrays such that for every $m, n, l \in \omega$, either $T_{0}$ does not diagonalize against $\left(E_{n}, E_{n, m, l}\right)$ or $T_{1}$ does not diagonalize against $\left(F_{n, m}, F_{n, m, l}\right)$. The class $\mathcal{B}$ can be coded as a closed set in the Baire space $\omega^{\omega}$. By hypothesis, $\mathcal{B}$ has no $C$-computable member. By [13, Corollary 2.9], there is an $\vec{R}$-cohesive set $G$ such that $\mathcal{B}$ has no $C \oplus G$-computable member. By definition of $\mathcal{B},\left(T_{0}, T_{1}\right)$ is $C \oplus G$-4-hyperimmune.

We also need the preservation of 4-hyperimmunity of WKL.

Proposition 3.4. WKL preserves 4-hyperimmunity. i.e., For every set $C$ and $C$-4hyperimmune $\mathrm{EM}$ instance pair $\left(T_{0}, T_{1}\right)$, every nonempty $\Pi_{1}^{0, C}$ class $\mathcal{P} \subseteq 2^{\omega}$, there is a $G \in \mathcal{P}$ such that $\left(T_{0}, T_{1}\right)$ is $C \oplus G$-4-hyperimmune.

Proof. Assume $C=\emptyset$, and fix $\left(T_{0}, T_{1}\right)$ and a $\Pi_{1}^{0}$ class $\mathcal{P} \subseteq 2^{\omega}$. We will prove our proposition with a forcing with $\Pi_{1}^{0}$ non-empty subclasses of $\mathcal{P}$. We satisfy the 
requirement:

$$
\begin{aligned}
& \mathcal{R}_{e}: \text { If } \Phi_{e}^{G} \text { is total, then for some } n, m, l \in \omega, \\
& T_{0} \text { diagonalizes against }\left(\Phi_{e}^{G}(n ; 1), \Phi_{e}^{G}(n, m, l ; 2)\right) \text { and } \\
& T_{1} \text { diagonalizes against }\left(\Phi_{e}^{G}(n, m ; 3), \Phi_{e}^{G}(n, m, l ; 4)\right) .
\end{aligned}
$$

The core of the argument is the following lemma :

Lemma 3.5. For every index e, every condition $c$ admits an extension forcing $\mathcal{R}_{e}$.

Proof. Let $\mathcal{Q} \subseteq \mathcal{P}$ be a condition. We define a partial computable 4-array as follows.

Defining $U_{n}$. Given $n \in \omega$, search computably for some finite set $U_{n}>n$ such that for every $X \in \mathcal{Q}$,

$$
\Phi_{e}^{X}(n ; 1) \downarrow \subseteq U_{n} .
$$

Defining $V_{n, m}$. Given $n, m \in \omega$, search computably for some finite set $V_{n, m}>n$ such that for every $X \in \mathcal{Q}$,

$$
\Phi_{e}^{X}(n, m ; 2) \downarrow \subseteq V_{n, m}
$$

Defining $U_{n, m, l}, V_{n, m, l}$. Given $n, m, l \in \omega$, search computably for some finite sets $U_{n, m, l}>m, V_{n, m, l}>l$ such that for every $X \in \mathcal{Q}$,

$$
\Phi_{e}^{X}(n, m, l ; 3) \downarrow \subseteq U_{n, m, l} \wedge \Phi_{e}^{X}(n, m, l ; 4) \downarrow \subseteq V_{n, m, l} .
$$

We now have multiple outcomes, depending on which $U_{n}$ and $V_{n, m}$ is found.

- Case 1: $U_{n}$ is not found for some $n \in \omega$. Then by compactness, the $\Pi_{1}^{0}$ class $\mathcal{W}$ of $X \in \mathcal{Q}$ so that $\Phi_{e}^{X}(n ; 1) \uparrow$ is nonempty. Thus $\mathcal{W}$ is the desired extension.

- Case 2: $V_{n, m}$ is not found for some $n, m \in \omega$. Then by compactness, the $\Pi_{1}^{0}$ class $\mathcal{W}$ of $X \in \mathcal{Q}$ so that $\Phi_{e}^{X}(n, m ; 2) \uparrow$ is nonempty. Thus $\mathcal{W}$ is the desired extension.

- Case 3: $U_{n}$ or $V_{n, m}$ is not found for some $n, m \in \omega$. Then by compactness, the $\Pi_{1}^{0}$ class $\mathcal{W}$ of $X \in \mathcal{Q}$ so that

$$
\Phi_{e}^{X}(n, m, l ; 3) \uparrow \vee \Phi_{e}^{X}(n, m, l ; 4) \uparrow
$$

is nonempty. Thus $\mathcal{W}$ is the desired extension.

- Case 4: $U_{n}, V_{n, m}, U_{n, m, l}, V_{n, m, l}$ are found for every $n, m \in \omega$. Since $\left(T_{0}, T_{1}\right)$ is 4-hyperimmune, there exist $n, m, l$ such that $T_{0}, T_{1}$ diagonalizes against $\left(U_{n}, U_{n, m, l}\right)$ and $\left(V_{n, m}, V_{n, m, l}\right)$ respectively. Thus $\mathcal{Q}$ already forces $\mathcal{R}_{e}$.

Let $\mathcal{F}=\left\{\mathcal{P}_{0}, \mathcal{P}_{1}, \ldots\right\}$ be a sufficiently generic filter for this notion of forcing, where $c_{s}=\left(F_{s}, X_{s}\right)$, and let $G \in \bigcap_{s} \mathcal{P}_{s}$. In particular, $G \in \mathcal{P}$ and by Lemma 3.5. $\left(T_{0}, T_{1}\right)$ is $C \oplus G$-4-hyperimmune. This completes the proof of Theorem 3.4 .

The rest of this section will be dedicated to the proof of Theorem 3.6 from which Theorem 1.7 follows.

Theorem 3.6. If a pair of EM instance $\left(T_{0}, T_{1}\right)$ is $C$-4-hyperimmune, then for every $C$-computable EM instance $T$, there exists a solution $G$ to $T$ such that either $C \oplus G$ does not compute a solution to $T_{0}$, or $C \oplus G$ does not compute a solution to $T_{1}$. 
THE REVERSE MATHEMATICS OF THE THIN SET AND THE ERDŐS-MOSER THEOREM\&9

Proof. For notation convenience, we assume $C=\emptyset$. Fix $\left(T_{0}, T_{1}\right)$ and $T$ as in Theorem 3.6. By Proposition 3.3, we may assume that $T$ is stable (for every $x \in \omega$, either $x \rightarrow_{T} y$ for all but finitely many $y$, or $y \rightarrow_{T} x$ for all but finitely many $y$ ).

In the rest of the proof, every Turing functional $\Phi^{G}$ is computing a set of integers, namely $\left\{n: \Phi^{G}(n) \downarrow=1\right\}$; so it makes sense to write $\Phi^{G} \cap A$. Let $A_{0} \sqcup A_{1}$ be a 2-partition (of $\omega$ ) such that $x \in A_{0}$ if and only if $x \rightarrow_{T} y$ for all but finitely many $y \in \omega$ (which is well defined since $T$ is stable). This automatically ensures that $x \in A_{1}$ iff $y \rightarrow_{T} x$ for all but finitely many $y \in \omega$. For a set $Z \subseteq \omega$, a 2-partition $X_{0} \sqcup X_{1}$ of $\omega$, we say $Z$ is compatible with $X_{0} \sqcup X_{1}$ if $Z \cap X_{0} \rightarrow_{T} Z \cap X_{1}$. Note that if $Z \subseteq X_{i}$ for some $i$, then $Z$ is compatible with $X_{0} \sqcup X_{1}$.

A condition is a Mathias condition $(F, X)$ with the following properties:

(a) $F$ is $T$-transitive and compatible with $A_{0} \sqcup A_{1}$;

(b) $F \cap A_{0} \rightarrow_{T} X \rightarrow_{T} F \cap A_{1}$;

(c) $\left(T_{0}, T_{1}\right)$ is $X$-4-hyperimmune.

A precondition is a Mathias condition satisfying (a)(c).

Lemma 3.7. Let $(F, X)$ be a condition and $Y \subseteq X$.

(1) If $Y$ is T-transitive, then $F \cup Y$ is T-transitive.

(2) If $Y$ is compatible with $A_{0} \sqcup A_{1}$, then $F \cup Y$ is compatible with $A_{0} \sqcup A_{1}$.

(3) For every precondition $(E, Y)$, there is a $b \in \omega$ so that $(E, Y \cap(b, \infty))$ is a condition.

Proof. Item (1)(2) follows from property (b) of $(F, X)$. Item (3) follows from definition of $A_{0}, A_{1}$.

Lemma 3.8. For every condition $(F, X)$ there exists an extension $(E, Y)$ such that $|E|>|F|$.

Proof. Let $x \in X \backslash F$. Clearly $\{x\}$ is $T$-transitive and compatible with $A_{0} \sqcup A_{1}$. By Lemma 3.7 item (1) (2), $(F \cup\{x\}, X)$ is a precondition.

For $e \in \omega, i \in 2$, let $\mathcal{R}_{e}^{i}$ denote the requirement:

$$
\Phi_{e}^{G} \text { is not a solution to } T_{i} \text {. }
$$

We will construct a solution $G$ of $T$ satisfying:

$$
\mathcal{R}_{e_{0}, e_{1}}: \mathcal{R}_{e_{0}}^{0} \vee \mathcal{R}_{e_{1}}^{1}
$$

for all $e_{0}, e_{1} \in \omega$. A condition $(F, X)$ forces $\mathcal{R}_{e_{0}, e_{1}}$ if: for every solution $G$ of $T$ satisfying $(F, X), G$ satisfies $\mathcal{R}_{e_{0}, e_{1}}$. Note that the definition of forcing is slightly different from that in section 2, that we restrict on $T$-transitive set. This restriction cannot be applied in section 2 since there we deal with arbitrary instance instead of computable instance.

Lemma 3.9. For every condition $c$ and indices $e_{0}, e_{1} \in \omega$, there is an extension of c forcing $\mathcal{R}_{e_{0}, e_{1}}$.

Proof. Fix $c=(D, X)$. By Lemma 3.7 for notation convenience, we assume $D=\emptyset$ and $X=\omega$. We firstly describe a process to partially compute a 4 -array. Then we show that if the computation diverges, we obtain an extension $d \leq c$ forcing $\mathcal{R}_{e_{0}, e_{1}}$ in the $\Pi_{1}^{0}$ way: one of $\Phi_{e_{i}}^{G}$ is not infinite; and if the computation converges, we 
obtain $d \leq c$ forcing $\mathcal{R}_{e_{0}, e_{1}}$ in a $\Sigma_{1}^{0}$ way: for some $n, m, l$ so that $T_{0}, T_{1}$ diagonalizes against $\left(V_{n}, V_{n, m}\right),\left(U_{n, m}, U_{n, m, l}\right)$ respectively, either $\Phi_{e_{0}}^{G} \cap U_{n} \neq \emptyset \wedge \Phi_{e_{0}}^{G} \cap U_{n, m} \neq \emptyset$; or $\Phi_{e_{1}}^{G} \cap V_{n, m} \neq \emptyset \wedge \Phi_{e_{1}}^{G} \cap V_{n, m, l} \neq \emptyset$ (which means for some $i<2$, $\Phi_{e_{i}}^{G}$ is not a solution to $T_{i}$ ).

Defining $U_{n}$. Given $n \in \omega$, search computably for some finite set $U_{n}>n$ such that for every 8-partition $X_{0} \sqcup \cdots \sqcup X_{7}=\omega$, there exists an $i<8$, a finite $T$ transitive set $E \subseteq X_{i}$ such that $\Phi_{e_{0}}^{E} \cap U_{n} \neq \emptyset$.

Defining $U_{n, m}, V_{n, m}$. Given $n, m \in \omega$, search computably for some finite set $U_{n, m}>m, V_{n, m}>n$ such that for every 4-partition $X_{0} \sqcup \cdots \sqcup X_{3}$ :

(a) either there exists an $i<4$, a finite $T$-transitive set $E \subseteq X_{i}$ such that $\Phi_{e_{0}}^{E} \cap U_{n} \neq \emptyset$ and $\Phi_{e_{0}}^{E} \cap U_{n, m} \neq \emptyset$

(b) or there exist $j \neq i<4$ and two finite $T$-transitive sets $F \subseteq X_{j}, E \subseteq X_{i}$ such that $\Phi_{e_{0}}^{E} \cap U_{n} \neq \emptyset$ and $\Phi_{e_{1}}^{F} \cap V_{n, m} \neq \emptyset$.

Defining $U_{n, m, l}, V_{n, m, l}$. Given $n, m \in \omega$, search computably for some finite sets $U_{n, m, l}>m, V_{n, m, l}>l$ such that for every 2-partition $X_{0} \sqcup X_{1}$, there exists a finite $T$-transitive set $E$ compatible with $X_{0} \sqcup X_{1}$ such that:

(p) either $\Phi_{e_{0}}^{E} \cap U_{n} \neq \emptyset$ and $\Phi_{e_{0}}^{E} \cap U_{n, m, l} \neq \emptyset$;

(q) or $\Phi_{e_{1}}^{E} \cap V_{n, m} \neq \emptyset$ and $\Phi_{e_{1}}^{E} \cap V_{n, m, l} \neq \emptyset$.

Case 1: $U_{n}$ is not found for $n$.

This is straightforward. By compactness, the following $\Pi_{1}^{0}$ class $\mathcal{P}$ of 8-partitions $X_{0} \sqcup \cdots \sqcup X_{7}$ is nonempty: for every $i<8$, every $T$-transitive finite set $E \subseteq X_{i}$, $\Phi_{e_{0}}^{E} \cap(n, \infty)=\emptyset$. As WKL preserves 4-hyperimmunity (Proposition 3.4), there exists a member $X_{0} \sqcup \cdots \sqcup X_{7}$ of $\mathcal{P}$ so that $\left(T_{0}, T_{1}\right)$ is $\oplus_{i<8} X_{i}$-4-hyperimmune. Fix any $i<8$ such that $X_{i}$ is infinite. Then $\left(D, X_{i}\right)$ is an extension of $c$ forcing $\mathcal{R}_{e_{0}, e_{1}}$.

Case 2: $U_{n}$ is found but not $\left(U_{n, m}, V_{n, m}\right)$ for some $n, m$.

By compactness, the following $\Pi_{1}^{0}$ class $\mathcal{P}$ of 4-partitions $X_{0} \sqcup \cdots \sqcup X_{3}$ is nonempty:

(a) for every $i<4$, every finite $T$-transitive set $E \subseteq X_{i}$, we have $\Phi_{e_{0}}^{E} \cap U_{n}=$ $\emptyset \vee \Phi_{e_{0}}^{E} \cap(m, \infty)=\emptyset ;$ and

(b) for every $j \neq i<4$ and every two finite $T$-transitive sets $F \subseteq X_{j}, E \subseteq X_{i}$, we have $\Phi_{e_{0}}^{E} \cap U_{n}=\emptyset \vee \Phi_{e_{1}}^{F} \cap(n, \infty)=\emptyset$.

As WKL preserves 4-hyperimmunity (Proposition 3.4), there exists a member $X_{0} \sqcup \cdots \sqcup X_{3}$ of $\mathcal{P}$ so that $\left(T_{0}, T_{1}\right)$ is $\oplus_{i<4} X_{i}$-4-hyperimmune. Consider the 8partition $\left(X_{i} \cap A_{k}: i<4, k<2\right)$. By definition of $U_{n}$, there exist $i<4, k<2$ and a finite $T$-transitive set $E \subseteq X_{i} \cap A_{k}$, such that $\Phi_{e_{0}}^{E} \cap U_{n} \neq \emptyset$.

Subcase 1: $X_{i}$ is infinite.

Since $E \subseteq A_{k}, E$ is compatible with $A_{0} \sqcup A_{1}$. So $d=\left(E, X_{i}\right)$ is a precondition extending $c$. Note that by property (a) of $X_{0} \sqcup \cdots \sqcup X_{3}$, for every $T$-transitive set $G$ satisfying $d$ (so $G \subseteq X_{i}$ ), we have $\Phi_{e_{0}}^{G} \cap(m, \infty)=\emptyset$ (since $\Phi_{e_{0}}^{G} \cap U_{n} \neq \emptyset$ ). Thus, $d$ forces $\mathcal{R}_{e_{0}, e_{1}}$.

Subcase 2: $X_{i}$ is finite.

Then there exists a $j \neq i$ such that $X_{j}$ is infinite. Note that by property (b) of $X_{0} \sqcup \cdots \sqcup X_{3}$, for every $T$-transitive set $G \subseteq X_{j}$, we have $\Phi_{e_{1}}^{G} \cap(n, \infty)=\emptyset$. Thus the condition $\left(D, X_{j}\right)$ extends $c$ and forces $\mathcal{R}_{e_{0}, e_{1}}$. 
THE REVERSE MATHEMATICS OF THE THIN SET AND THE ERDŐS-MOSER THEOREM\$1

Case 3: $U_{n}, U_{n, m}, V_{n, m}$ are found but not $\left(U_{n, m, l}, V_{n, m, l}\right)$ for some $n, m, l \in \omega$.

By compactness, the following $\Pi_{1}^{0}$ class $\mathcal{P}$ of 2-partitions $X_{0} \sqcup X_{1}$ is nonempty: for every finite $T$-transitive finite set $E$ compatible with $X_{0} \sqcup X_{1}$, we have

(p) $\Phi_{e_{0}}^{E} \cap U_{n}=\emptyset \vee \Phi_{e_{0}}^{E} \cap(m, \infty)=\emptyset$; and

(q) $\Phi_{e_{1}}^{E} \cap V_{n, m}=\emptyset \vee \Phi_{e_{1}}^{E} \cap(l, \infty)=\emptyset$.

By Proposition 3.4 there exists a member $X_{0} \sqcup X_{1}$ of $\mathcal{P}$ so that $\left(T_{0}, T_{1}\right)$ is $\oplus_{j<2} X_{j}$-4-hyperimmune. Consider the 4-partition $\left(X_{j} \cap A_{k}: j, k<2\right)$. By property (p) of $X_{0} \sqcup X_{1}$, for every $j, k<2$ and $E \subseteq X_{j} \cap A_{k}$ (so $E$ is compatible with $X_{0} \sqcup X_{1}$ ),

$$
\Phi_{e_{0}}^{E} \cap U_{n}=\emptyset \vee \Phi_{e_{0}}^{E} \cap(m, \infty)=\emptyset .
$$

Combine with the definition of $U_{n, m}, V_{n, m}$ (where we take the 4-partition to be $\left(X_{j} \cap A_{k}: j, k<2\right)$ and note that property (a) there does not occur so property (b) occurs), we have: there are $(j, k) \neq(\hat{j}, \hat{k})$ and finite $T$-transitive sets $E \subseteq$ $X_{j} \cap A_{k}, F \subseteq X_{\hat{j}} \cap A_{\hat{k}}$ such that

$$
\Phi_{e_{0}}^{E} \cap U_{n}, \Phi_{e_{1}}^{F} \cap V_{n, m} \neq \emptyset .
$$

Subcase 1: Either $X_{j}$ or $X_{\hat{j}}$ is infinite.

Suppose $X_{j}$ is infinite. Consider the precondition $d=\left(E, X_{j}\right)$ extending $c$. Note that for every $T$-transitive set $G$ satisfying $d, G$ is compatible with $X_{0} \sqcup X_{1}$ (since $G \subseteq X_{j}$ ). Thus by property (p) of $X_{0} \sqcup X_{1}$ and $\Phi_{e_{0}}^{E} \cap U_{n} \neq \emptyset$, for every $T$-transitive set $G$ satisfying $d$, we have $\Phi_{e_{0}}^{G} \cap(m, \infty)=\emptyset$. Thus $d$ forces $\mathcal{R}_{e_{0}, e_{1}}$. Suppose now $X_{\hat{j}}$ is infinite. Then, taking $d=\left(F, X_{\hat{j}}\right)$, a similar argument shows that by property (q) of $X_{0} \sqcup X_{1}$, for every $T$-transitive set $G$ satisfying $d$, we have $\Phi_{e_{1}}^{G} \cap(l, \infty)=\emptyset$. Thus $d$ forces $\mathcal{R}_{e_{0}, e_{1}}$. Thus we are done in this subcase.

Subcase 2: Both $X_{j}, X_{\hat{j}}$ are finite.

This implies $j=\hat{j}$ since $X_{0} \sqcup X_{1}=\omega$, and then $k \neq \hat{k}$ since $(j, k) \neq(\hat{j}, \hat{k})$. Let $b$ be sufficiently large to witness the limits of the elements of $F$ and $E$ with respect to the tournament.

If $j=\hat{j}=0$ (so $X_{1}$ is infinite) and $\hat{k}=0, k=1$, consider the condition $d=\left(F, X_{1} \backslash[0, b]\right)$. Since $F \subseteq A_{\hat{k}}=A_{0}$, we have $F \rightarrow_{T} X_{1} \backslash[0, b]$. Therefore for every $G \subseteq X_{1} \backslash[0, b], F \cup G$ is compatible with $X_{0} \sqcup X_{1}$ (since $F \subseteq X_{0}$ ). Thus for every $T$-transitive set $G$ satisfying $d$, by property (q) of $X_{0} \sqcup X_{1}$ and since $\Phi_{e_{1}}^{F} \cap V_{n, m} \neq \emptyset$, we have $\Phi_{e_{1}}^{G} \cap(l, \infty)=\emptyset$. Thus $d$ forces $\mathcal{R}_{e_{1}}^{1}$.

If $j=\hat{j}=1$ (so $X_{0}$ is infinite) and $\hat{k}=0, k=1$, consider the condition $d=\left(E, X_{0} \backslash[0, b]\right)$. Since $E \subseteq A_{k}=A_{1}$, we have $X_{0} \backslash[0, b] \rightarrow_{T} E$. Therefore for every set $G \subseteq X_{0} \backslash[0, b], E \cup G$ is compatible with $X_{0} \sqcup X_{1}$ (since $E \subseteq X_{1}$ ). Thus for every $T$-transitive set $G$ satisfying $d$, by property (p) of $X_{0} \sqcup X_{1}$ and $\Phi_{e_{0}}^{E} \cap U_{n} \neq \emptyset$, we have $\Phi_{e_{0}}^{G} \cap(m, \infty)=\emptyset$. Thus $d$ forces $\mathcal{R}_{e_{0}}^{0}$.

If $j=\hat{j}=0$ (so $X_{1}$ is infinite) and $\hat{k}=1, k=0$, then the condition $\left(E, X_{1} \backslash[0, b]\right.$ ) forces $\mathcal{R}_{e_{0}}^{0}$ by a similar argument using property (p) of $X_{0} \sqcup X_{1}$.

If $j=\hat{j}=1$ (so $X_{0}$ is infinite) and $\hat{k}=1, k=0$, then the condition $\left(F, X_{0} \backslash[0, b]\right)$ forces $\mathcal{R}_{e_{1}}^{1}$ by a similar argument using property (q) of $X_{0} \sqcup X_{1}$. 
Case 4: $U_{n}, U_{n, m}, V_{n, m}, U_{n, m, l}, V_{n, m, l}$ is found for all $n, m, l$.

Since $\left(T_{0}, T_{1}\right)$ is 4-hyperimmune, there exist $n, m, l$ such that $T_{0}, T_{1}$ diagonalizes against $\left(U_{n}, U_{n, m, l}\right)$ and $\left(V_{n, m}, V_{n, m, l}\right)$, respectively. By definition of $U_{n, m, l}, V_{n, m, l}$ (where we take $X_{0} \sqcup X_{1}$ to be $A_{0} \sqcup A_{1}$ ), there exists a finite $T$-transitive set $F$ compatible with $A_{0} \sqcup A_{1}$ such that

(p) either $\Phi_{e_{0}}^{F} \cap U_{n} \neq \emptyset$ and $\Phi_{e_{0}}^{F} \cap U_{n, m, l} \neq \emptyset$;

(q) or $\Phi_{e_{1}}^{F} \cap V_{n, m} \neq \emptyset$ and $\Phi_{e_{1}}^{F} \cap V_{n, m, l} \neq \emptyset$.

Let $d=(F, X)$. We claim that $d$ forces $\mathcal{R}_{e_{0}, e_{1}}$ by forcing $\mathcal{R}_{e_{0}}^{0}$ on case (p) and $\mathcal{R}_{e_{1}}^{1}$ on case (q). Let $G$ be a set satisfying $d$. In the case (p), $\Phi_{e_{0}}^{G}$ has a non-empty intersection with both $U_{n}$ and $U_{n, m, l}$, in which case $\Phi_{e_{0}}^{G}$ is not a solution to $T_{0}$ (recall the definition of diagonal against); and in the case (q), $\Phi_{e_{1}}^{G}$ has non-empty intersection with both $V_{n, m}$ and $V_{n, m, l}$, in which case $\Phi_{e_{1}}^{G}$ is not a solution to $T_{1}$. This completes the proof of the lemma.

Let $\mathcal{F}=\left\{c_{0}, c_{1}, \ldots\right\}$ be a sufficiently generic filter for this notion of forcing, where $c_{s}=\left(F_{s}, X_{s}\right)$, and let $G=\bigcup_{s} F_{s}$. By property (a) of a condition, $G$ is $T$ transitive. By Lemma 3.8, $G$ is infinite, and by Lemma 3.9, $G$ satisfies $\mathcal{R}_{e_{0}, e_{1}}$ for all $e_{0}, e_{1}$. By pairing argument, this means either $G$ does not compute a solution to $T_{0}$, or $G$ does not compute a solution to $T_{1}$. This completes the proof of Theorem 3.6 .

\section{ACKNOWLEDGEMENTS}

The second author was partially supported by grant ANR "ACTC" \#ANR-19CE48-0012-01.

\section{REFERENCES}

[1] Andrey Bovykin and Andreas Weiermann. The strength of infinitary Ramseyan principles can be accessed by their densities. Annals of Pure and Applied Logic, page 4, 2005. To appear.

[2] Peter A. Cholak, Mariagnese Giusto, Jeffry L. Hirst, and Carl G. Jockusch Jr. Free sets and reverse mathematics. Reverse mathematics, 21:104-119, 2001.

[3] Harvey M. Friedman. Fom:53:free sets and reverse math and fom:54:recursion theory and dynamics. Available at https://www.cs.nyu.edu/pipermail/ fom/

[4] Harvey M. Friedman. Some systems of second order arithmetic and their use. In Proceedings of the International Congress of Mathematicians, Vancouver, volume 1, pages 235-242. Canadian Mathematical Society, Montreal, Quebec, 1974.

[5] Denis R. Hirschfeldt and Richard A. Shore. Combinatorial principles weaker than Ramsey's theorem for pairs. Journal of Symbolic Logic, 72(1):171-206, 2007.

[6] Carl G. Jockusch. Ramsey's theorem and recursion theory. Journal of Symbolic Logic, 37(2):268-280, 1972.

[7] Carl G. Jockusch and Robert I. Soare. $\Pi_{1}^{0}$ classes and degrees of theories. Transactions of the American Mathematical Society, 173:33-56, 1972.

[8] Manuel Lerman, Reed Solomon, and Henry Towsner. Separating principles below Ramsey's theorem for pairs. Journal of Mathematical Logic, 13(02):1350007, 2013. 
THE REVERSE MATHEMATICS OF THE THIN SET AND THE ERDŐS-MOSER THEOREM\$3

[9] Lu Liu. RT 2 does not imply $\mathrm{WKL}_{0}$. Journal of Symbolic Logic, 77(2):609-620, 2012.

[10] Lu Liu. Cone avoiding closed sets. Transactions of the American Mathematical Society, 367(3):1609-1630, 2015.

[11] Antonio Montalbán. Open questions in reverse mathematics. Bulletin of Symbolic Logic, 17(03):431-454, 2011.

[12] Piergiorgio Odifreddi. Classical recursion theory: The theory of functions and sets of natural numbers. Elsevier, 1992.

[13] Ludovic Patey. Combinatorial weaknesses of Ramseyan principles. In preparation. Available at http://ludovicpatey.com/media/research/ combinatorial-weaknesses-draft.pdf, 2015.

[14] Ludovic Patey. Degrees bounding principles and universal instances in reverse mathematics. Annals of Pure and Applied Logic, 166(11):1165-1185, 2015.

[15] Ludovic Patey. Open questions about Ramsey-type statements in reverse mathematics. Bulletin of Symbolic Logic, 2015. To appear. Available at http://arxiv.org/abs/1506.04780.

[16] Ludovic Patey. Partial orders and immunity in reverse mathematics. 2016.

[17] Ludovic Patey. The reverse mathematics of Ramsey-type theorems. PhD thesis, Université Paris Diderot, 2016.

[18] Ludovic Patey. The weakness of being cohesive, thin or free in reverse mathematics. Israel J. Math., 216(2):905-955, 2016.

[19] David Seetapun and Theodore A. Slaman. On the strength of Ramsey's theorem. Notre Dame Journal of Formal Logic, 36(4):570-582, 1995.

[20] Stephen G. Simpson. Subsystems of Second Order Arithmetic. Cambridge University Press, 2009.

[21] Wei Wang. Some logically weak Ramseyan theorems. Advances in Mathematics, 261:1-25, 2014.

Department of Mathematics, Central South University, ChangSha 410083, People's Republic of China

Email address: g.jiayi.liu@gmail.com

CNRS, Institut Camille Jordan, Université Claude Bernard Lyon 1, 43 boulevard du 11 novembre 1918, F-69622 Villeurbanne Cedex, France

Email address: ludovic.patey@computability.fr 